\title{
Frictional Contact Problems for Thin Elastic Structures and Weak Solutions of Sweeping Processes
}

\author{
PATRICK BALLARD
}

\begin{abstract}
The linearized equilibrium equations for straight elastic strings, beams, membranes or plates do not couple tangential and normal components. In the quasi-static evolution occurring above a fixed rigid obstacle with Coulomb dry friction, the normal displacement is governed by a variational inequality, whereas the tangential displacement is seen to obey a sweeping process, the theory of which was extensively developed by Moreau in the 1970s. In some cases, the underlying moving convex set has bounded retraction and, in these cases, the sweeping process can be solved by directly applying Moreau's results. However, in many other cases, the bounded retraction condition is not fulfilled and this is seen to be connected to the possible event of moving velocity discontinuities. In such a case, there are no strong solutions and we have to cope with weak solutions of the underlying sweeping process.
\end{abstract}

\section{Motivation and outline}

\subsection{Background}

The frictionless equilibrium of linearly elastic strings and beams (or membranes and plates) above a fixed rigid obstacle provides an archetypical example of variational inequality, the theory of which was extensively developed in the 1970s. This paper deals with the situation where Coulomb dry friction between the elastic structure and the obstacle should be assumed to occur in addition. More specifically, it focuses here on cases where the linearized equilibrium equation can be used and consider the quasi-static evolution problem given by the usual Coulomb friction law. Surprisingly, this seems to be the first time this class of problems has been investigated. One specific (and comfortable) feature of these problems is the fact that the linearized equilibrium equations do not couple the normal and tangential components of the displacement. The problem that governs the normal displacement is, therefore, the same as that arising in the frictionless situation, that is, a 
variational inequality at every instant. Solving this variational inequality at every instant gives the normal component of the reaction force exerted by the obstacle and therefore gives the threshold for the friction law, which generally depends on the time and the position. The evolution problem that governs the tangential displacement is shown to provide an archetypical example of a sweeping process in a Hilbert space, the theory of which was developed in the seventies by MoREAU [10] with a view to applying it to elastoplastic systems.

The fact that the linearized equilibrium equations do not couple the normal and tangential components of the displacement lend the situation under consideration some similarity to perfect plasticity. Also, the moving tangential velocity discontinuities that will be exhibited in this paper should certainly be brought alongside the velocity discontinuities that are well known to spontaneously occur in perfect plasticity [11].

This uncoupling is a specific feature of the straight thin elastic structures that are the only ones considered in this paper. The situation is rather different in the more usual situation where a massive elastic body is considered. Indeed, in that case, the linearized equilibrium equations couple normal and tangential components so that monotonicity is lost. This raises important mathematical difficulties in the analysis. An existence result for the corresponding evolution problem (quasi-static contact problem in linear elasticity with Coulomb friction) was obtained only in 2000 by ANDERSSON [2] using the approach developed in the pioneering work of JARUŠEK [7]. Very little is known about uniqueness, but the lack of monotonicity makes the situation tricky [3]. For a recent survey on the analysis of frictional contact problems for massive bodies, the reader is referred to [6].

\subsection{The basic evolution problem}

Let us consider a straight elastic string which is uniformly tensed in its reference configuration and an orthonormal basis $\left(\mathbf{e}_{x}, \mathbf{e}_{y}\right)$ with $\mathbf{e}_{x}$ chosen along the direction of the string. A fixed rigid obstacle is described by the function $y=\psi(x)$. The string is loaded with a given body force $f \mathbf{e}_{x}+g \mathbf{e}_{y}$ and displacements $u_{0}^{\mathrm{p}} \mathbf{e}_{x}+v_{0}^{\mathrm{p}} \mathbf{e}_{y}$, $u_{1}^{\mathrm{p}} \mathbf{e}_{x}+v_{1}^{\mathrm{p}} \mathbf{e}_{y}$ are prescribed at extremities $x=0,1$. Let $u \mathbf{e}_{x}+v \mathbf{e}_{y}$ denote the displacement field in the string and $r \mathbf{e}_{x}+s \mathbf{e}_{y}$ denote the reaction force exerted by the obstacle on the string. Assuming that the linearized equilibrium equations can be used, one finds that the quasi-static evolution of that string above the obstacle with unilateral contact condition and Coulomb dry friction during the time interval $\left[t_{0}, T\right]$ is governed by

$$
\mid \begin{array}{ll}
u^{\prime \prime}+f+r=0, & \text { in }] 0,1\left[\times\left[t_{0}, T\right],\right. \\
r(\hat{u}-\dot{u})+\mu s(|\hat{u}|-|\dot{u}|) \geqq 0, \quad \forall \hat{u} \in \mathbb{R}, & \text { in }] 0,1\left[\times\left[t_{0}, T\right],\right. \\
u(0)=u_{0}^{\mathrm{p}}, \quad u(1)=u_{1}^{\mathrm{p}}, & \text { on }\left[t_{0}, T\right], \\
v^{\prime \prime}+g+s=0, & \text { in }] 0,1[, \\
v-\psi \geqq 0, \quad s \geqq 0, \quad s(v-\psi) \equiv 0, & \text { in }] 0,1\left[\times\left[t_{0}, T\right],\right. \\
v(0)=v_{0}^{\mathrm{p}}, \quad v(1)=v_{1}^{\mathrm{p}}, & \text { on }\left[t_{0}, T\right] .
\end{array}
$$

where $\mu$ is the friction coefficient, which is assumed to be given. 
The last three lines of system (1) govern the normal component $v$ of the displacement, and are not coupled with the other equations of system (1). Therefore, at every instant, $v$ obeys the same variational inequality as that governing the more usual frictionless situation. Assuming that this problem has been solved, the normal component $s$ of the reaction is now supposed to be given in the study of the tangential problem, that is, the first three lines of system (1). It is necessary of course to know what regularity $s$ can be expected to show, and this question requires detailed analysis of the normal problem governed by the variational inequality. As we will see, the regularity of $s$ is crucial to the analysis of the tangential problem.

Introducing for every $t \in\left[t_{0}, T\right]$, the closed, convex subset of $H^{1}(0,1 ; \mathbb{R})$ defined by

$$
\begin{aligned}
& \mathcal{C}(t)=\left\{u \in H^{1} \mid u(x=0)=u_{0}^{\mathrm{p}}, \quad u(x=1)=u_{1}^{\mathrm{p}},\right. \\
& \left.\quad \text { and } \forall \varphi \in H_{0}^{1}, \quad\left\langle u^{\prime \prime}+f, \varphi\right\rangle_{H^{-1}, H_{0}^{1}} \leqq\langle\mu s,|\varphi|\rangle_{H^{-1}, H_{0}^{1}}\right\},
\end{aligned}
$$

and equipping $H^{1}$ with the scalar product

$$
(\varphi \mid \psi)_{H^{1}}=\int_{0}^{1} \bar{\varphi}^{\prime}(x) \bar{\psi}^{\prime}(x) \mathrm{dx}+\varphi(0) \psi(0)+\varphi(1) \psi(1),
$$

taking

$$
\bar{\varphi}(x)=\varphi(x)-\varphi(0)-x(\varphi(1)-\varphi(0)) \in H_{0}^{1},
$$

the evolution problem that governs the tangential displacement $u$ can be written in the following concise form:

$$
-\dot{u}(t) \in \partial I_{\mathcal{C}(t)}[u(t)],
$$

after eliminating the unknown reaction force $r$ (see section 4 for details). In this differential inclusion, $I_{\mathcal{C}(t)}[\cdot]$ denotes the indicatrix function of $\mathcal{C}(t)$ (which equals 0 at any point of $\mathcal{C}(t)$ and $+\infty$ elsewhere), and $\partial I_{\mathcal{C}(t)}[\cdot]$ its subdifferential in the sense of the above scalar product in $H^{1}$, that is, the cone of all the outward normals to $\mathcal{C}(t)$ (which is empty at any point not belonging to $\mathcal{C}(t)$, and reduces to $\{0\}$ at an interior point, if any).

\subsection{Weak solutions of sweeping processes}

Let $H$ be a Hilbert space and $\mathcal{C}(t)$ a set-valued mapping defined on a time interval $\left[t_{0}, T\right]$ and the values of which are closed, convex and nonempty. A sweeping process is the evolution problem defined by

$$
\mid \begin{aligned}
& -\dot{u}(t) \in \partial I_{\mathcal{C}(t)}[u(t)], \quad \text { in }\left[t_{0}, T\right], \\
& u\left(t_{0}\right)=u_{0},
\end{aligned}
$$

with the given initial condition $u_{0} \in \mathcal{C}\left(t_{0}\right)$. This abstract evolution problem was introduced and studied by Jean Jacques MorEAU [10] with a view to using it in the 
analysis of elastoplastic systems. In kinematic terms, $\mathcal{C}(t)$ is a moving convex set and $u(t)$ a point in that set $\left(u(t) \in \mathcal{C}(t)\right.$ since $\partial I_{\mathcal{C}(t)}[\cdot]$ is empty at any point which does not belong to $\mathcal{C}(t)$ ). The evolution problem under consideration, therefore, has a geometrical interpretation, which is especially clear if $\mathcal{C}(t)$ has a non-empty interior. Indeed, whenever $u(t)$ is an interior point, $\partial I_{\mathcal{C}(t)}[u(t)]$ reduces to $\{0\}$ and the point $u(t)$ must remain at rest until meeting the boundary of $\mathcal{C}(t)$. It then proceeds in an inward normal direction, as if it were pushed by the boundary so as to go on belonging to $\mathcal{C}(t)$. The name "sweeping process", which was coined by Jean Jacques Moreau, refers to this vivid mechanical interpretation.

To discuss the existence of solutions to the sweeping process, some regularity assumptions about the set-valued mapping $\mathcal{C}(t)$ must be made. Actually, regularity is needed only when the set retracts, thus effectively sweeping the point $u(t)$. Jean Jacques Moreau defined and extensively studied the class of set-valued mappings $\mathcal{C}(t)$ with bounded retraction (see [9] or appendix A). In particular, set-valued mappings $\mathcal{C}(t)$ with bounded retraction admit a left limit $\mathcal{C}(t-)$, in the sense of Kuratowski (see appendix A), at any $\left.t \in] t_{0}, T\right]$ and a right limit $\mathcal{C}(t+)$ at any $t \in\left[t_{0}, T[\right.$.

Taking an arbitrary subdivision $P$ (finite partition into intervals of any sort) of $\left[t_{0}, T\right]$ and denoting by $I_{i}$ the corresponding intervals (which are indexed according to their successive order) with origin $t_{i}$ (left extremity, which does not necessarily belong to $I_{i}$ ), one can build the piecewise constant set-valued mapping $\mathcal{C}_{P}$ with closed convex values by using the following definition:

$$
\mathcal{C}_{P}\left(I_{i}\right)=\mathcal{C}_{i}=\mid \begin{array}{ll}
\mathcal{C}\left(t_{i}\right) & \text { if } t_{i} \in I_{i} \\
\mathcal{C}\left(t_{i}+\right) & \text { if } t_{i} \notin I_{i}
\end{array}
$$

Given the initial condition $u_{0} \in \mathcal{C}\left(t_{0}\right)$, the "catching-up algorithm" is based on the inductive projections given by

$$
u_{i+1}=\operatorname{proj}\left(u_{i}, \mathcal{C}_{i+1}\right)
$$

to build a step function $u_{P}:\left[t_{0}, T\right] \rightarrow H$, defined by

$$
u_{P}\left(I_{i}\right)=u_{i}
$$

This is simply a version of the implicit Euler algorithm for ordinary differential equations adapted to the differential inclusion involved. Assuming that $\mathcal{C}(t)$ has bounded retraction, MoREAU [10] proved that the net $u_{P}(P$ covering all the subdivisions of $\left.\left[t_{0}, T\right]\right)$ converges strongly in $H$, uniformly on $t \in\left[t_{0}, T\right]$, towards a function $u:\left[t_{0}, T\right] \rightarrow H$, which Moreau calls $a$ weak solution of the sweeping process. He then proved that this weak solution $u:\left[t_{0}, T\right] \rightarrow H$ has bounded variation and solves the sweeping process in the sense of "differential measures" (see [10] or appendix B). If $\mathcal{C}(t)$ has not only bounded retraction, but absolutely continuous retraction, it turns out that the weak solution $u:\left[t_{0}, T\right] \rightarrow H$ is absolutely continuous and is a strong solution of the sweeping process, that is

$$
-\dot{u}(t) \in \partial I_{\mathcal{C}(t)}[u(t)], \text { for a. a. } t \in\left[t_{0}, T\right] .
$$


The quasi-static evolution of the elastic string above the rigid obstacle when Coulomb friction is taken into account provides some natural examples of sweeping processes in the Hilbert space $H=H^{1}$. Some of these examples will be given in this paper, in cases where the underlying sweeping process has bounded retraction and Moreau's theory provides a unique weak solution which is also a solution in the sense of differential measures. In some of these examples, this solution also turns out to be a strong solution, but this is not always the case. More interestingly, it is easy to design an evolution problem for the elastic string where the underlying sweeping process turns out not to have bounded retraction. Sticking to the standpoint of the numerical computations, such examples require an extension of the definition of weak solutions for sweeping processes to a more general class of set-valued mappings $\mathcal{C}(t)$ than that of bounded retraction. Since the catching-up algorithm requires the existence of a right limit $\mathcal{C}(t+)$ in the sense of Kuratowski, it turns out that the class of $\mathcal{C}(t)$, which is suitable for defining weak solutions of sweeping processes in general, seems to be the class of so-called Wijsman-regulated set-valued mappings which is exactly the class of those $\mathcal{C}(t)$ with closed convex values that admit a left limit $\mathcal{C}(t-)$, in the sense of Kuratowski, at any $\left.t \in] t_{0}, T\right]$, and a right limit $\mathcal{C}(t+)$ at any $t \in\left[t_{0}, T\right.$. Wijsman-regulated $\mathcal{C}(t)$ are also characterized by the condition that for every $x \in H$, the function:

$$
t \mapsto \operatorname{proj}[x ; C(t)]
$$

is regulated (that is, is the uniform limit of a sequence of step functions, or, equivalently, admits a left and a right limit at every $t$ ). The name given to this class of set-valued mappings originates from the fact that the class of all closed nonempty subsets of $H$ can be equipped with a complete metrizable topology called the Wijsman topology. This is the weakest topology generated by the set functions $C \rightarrow d(x, C)$ when $x$ covers $H$ (here $d(x, C)$ denotes the distance of the point $x$ to the set $C$ ). Wijsman-regulated $\mathcal{C}(t)$ are exactly those set-valued mappings that are regulated in the sense defined by the Wijsman topology on the class of all closed non-empty subsets in $H$.

Weak solutions of sweeping processes associated with Wijsman-regulated $\mathcal{C}(t)$, when they exist, are proved to enjoy the same general properties as those established by Moreau in the case of weak solutions of sweeping processes based on $\mathcal{C}(t)$ with bounded retraction. Some examples of weak solutions of sweeping processes based on Wijsman-regulated $\mathcal{C}(t)$ that do not have bounded retraction are displayed in this paper. As we will see, these weak solutions do not necessarily have bounded variation. Examples will also be given of sweeping processes based on Wijsman-regulated $\mathcal{C}(t)$ that do not have any weak solution at all.

\subsection{Frictional contact problems for the elastic string}

Recalling that the tangential displacement of elastic strings obeys a sweeping process based on the set-valued mapping (2), a sufficient condition for $\mathcal{C}(t)$ to have 
bounded retraction is proved to be:

$$
\begin{aligned}
u_{0}^{\mathrm{p}}, u_{1}^{\mathrm{p}} & \in B V\left(\left[t_{0}, T\right] ; \mathbb{R}\right), \\
f & \in B V\left(\left[t_{0}, T\right] ; H^{-1}\right), \\
s & \in B V\left(\left[t_{0}, T\right] ; \mathcal{M}\right) .
\end{aligned}
$$

Here, $B V$ stands for "Bounded Variation" and $\mathcal{M}$ denotes the Banach space of the Radon measures on $[0,1]$, that is, the topological dual of $C^{0}([0,1] ; \mathbb{R})$. The first two lines in (3) give regularity assumptions about the data involved in the evolution problem, but the last line refers to the regularity of the solution of the normal problem governed by the variational inequality and therefore can not be controlled directly. It may occur that these regularity conditions are met and a detailed example is discussed in Section 4.3. In such a case, Moreau's results provide a unique solution:

$$
u \in B V\left(\left[t_{0}, T\right] ; H^{1}\right),
$$

and if the regularity that is met with the data is not only that of functions with "bounded variation" in time, but that of "absolutely continuous" functions, then the same will be true of $u$, which is a strong solution of the sweeping process. In such a circumstance, the tangential velocity $\dot{u}$ will belong to $H^{1}(0,1 ; \mathbb{R})$, at almost all values of $t \in\left[t_{0}, T\right]$, and will therefore be spatially continuous.

However, it may occur that the condition $s \in B V\left(\left[t_{0}, T\right] ; \mathcal{M}\right)$ is not fulfilled. A simple example of this occurrence is that of a string with a reference configuration lying on a rectilinear rigid obstacle (see Fig. 2). The data of the evolution problem are defined by $u_{0}^{\mathrm{p}}=v_{0}^{\mathrm{p}}=v_{1}^{\mathrm{p}} \equiv 0, u_{1}^{\mathrm{p}}$ is the function which takes the value 0 at $t=0$ and 1 at every $t>0$, and $f=\delta_{x=1 / 2-t}, g \equiv 0$ (the body force is a "moving transverse punctual force"). The unique solution of the normal problem is given by $v \equiv 0$, which entails $s \equiv-f$. Since for all $\left.t_{1}<t_{2} \in\right] 0,1[$,

$$
\left\|\delta_{t_{2}}-\delta_{t_{1}}\right\|_{\mathcal{M}}=2
$$

the normal reaction $s:\left[t_{0}, T\right] \rightarrow \mathcal{M}$ is neither a function with bounded variation nor a continuous function. Assuming for the sake of convenience that $\mu>2$, one still can arbitrarily subdivide the time interval $[0,1 / 3]$ and perform the successive projections of Moreau's catching-up algorithm. It then can be seen that the corresponding approximating step functions $u_{P}$ converge strongly in $H^{1}$, uniformly with respect to $t \in[0,1 / 3]$, towards the following function:

$$
u(x, t)=\mid \begin{array}{ll}
0, & \text { if } 0 \leqq x \leqq 1 / 2-t \\
\frac{x+t-1 / 2}{t+1 / 2}, & \text { if } 1 / 2-t \leqq x \leqq 1
\end{array}
$$

The graph of this function, together with that of the velocity $\dot{u}$, is plotted in Fig. 3 . The velocity can be seen to show a moving discontinuity; therefore, it does not belong to $H^{1}$ at any $t$. Consequently, the underlying $\mathcal{C}(t)$ does not have bounded retraction; however, it is Wijsman-regulated and the function $u$ is a weak solution of 
the underlying sweeping process (in the sense of the Definition 9 in Appendix B). It is worth noting that since the velocity is discontinuous, its value at the point of the string just below the load is not defined. One therefore cannot check if the Coulomb friction law is satisfied by the solution in the strong sense (that is, pointwise). The picture looks like that of perfect plasticity [11] where the spontaneous occurrence of velocity discontinuities requires to cope with weak solutions only. Extending Moreau's definition of weak solutions for sweeping processes to the case of Wijsman-regulated set-valued mappings leads to the appropriate definition of what should be called a weak solution of the frictional contact problem. This definition sticks to the standpoint of computational approximations. Another approach would consist of using a regularization procedure. A natural regularization method which could be used in the example under consideration would consist of "spreading out" the moving load a little bit by performing a spatial convolution. For example, the Dirac measure at $x$ can be approximated by the function taking the value $1 /(2 \varepsilon)$ at ] $x-\varepsilon, x+\varepsilon$ [ and 0 elsewhere. This suffices for the underlying $\mathcal{C}_{\varepsilon}(t)$ to have bounded retraction. The unique solution $u_{\varepsilon}$ of the corresponding sweeping process is given explicitly in Section 4.4. It can therefore be seen that $u_{\varepsilon}$ converges strongly in $H^{1}$ uniformly with respect to $t \in[0,1 / 3]$, towards the previously calculated weak solution $u$.

\subsection{Replacing the string by a beam}

Replacing the string by an elastic beam in the evolution problem (1) requires changing only the last three lines governing the normal displacement $v$, whereas the tangential problem governed by the first three lines remains unchanged. In particular, the equilibrium equation satisfied by $v$ is now an equation of order 4 . The normal component $s$ of the reaction force, which is now obtained after solving a variational inequality associated with the biharmonic operator, is therefore seen to be possibly a "moving Dirac measure" even in cases where all the data of the normal problem are $C^{\infty}$ in space and time. This means that moving tangential velocity discontinuities should generically be expected to occur in the case of the beam, and the underlying sweeping process should be expected to admit only weak solutions, even when arbitrarily smooth data are involved.

In this paper, it is proved that it suffices to require that the data,

$$
\begin{aligned}
u_{0}^{\mathrm{p}}, u_{1}^{\mathrm{p}}, v_{0}^{\mathrm{p}}, v_{1}^{\mathrm{p}}:\left[t_{0}, T\right] & \rightarrow \mathbb{R}, \\
f, g:\left[t_{0}, T\right] & \rightarrow H^{-1},
\end{aligned}
$$

should be regulated functions (that is, are the uniform limit of a sequence of step functions, or, equivalently, admit a left and a right limit at every $t$ ) to ensure that the moving set $\mathcal{C}(t)$ associated with the sweeping process governing the tangential problem will be Wijsman-regulated, so as to be able to speak about possible weak solutions. This claim, which relies on regularity analysis on the variational inequalities associated with the harmonic and biharmonic operators, holds true for strings as well as for beams.

However, these regularity assumptions are too weak to systematically ensure the existence of a weak solution to the underlying sweeping process. An example is 
provided that shows, in particular, that sweeping processes based on Wijsman-regulated set-valued mappings need not have weak solutions. The question as to what regularity assumptions about the data should be required to ensure the existence of a weak solution to the frictional contact problem is left open in this paper.

\section{Statement of the evolution problem for an elastic string}

The orthonormal basis $\left(\mathbf{e}_{x}, \mathbf{e}_{y}\right)$ will be used here in the affine Euclidean plane. Let us consider a string having the segment $[0,1] \times\{0\}$ as its reference configuration. This configuration undergoes some homogeneous tension $T_{0}>0$ and is an equilibrium configuration when the string is free of body forces.

Next, let us consider the given external body force,

$$
f \mathbf{e}_{x}+g \mathbf{e}_{y}
$$

Taking

$$
u \mathbf{e}_{x}+v \mathbf{e}_{y},
$$

to denote the displacement field in the string, one finds that the linearized equations that govern the equilibrium of the string, which is assumed to be elastic with stiffness $k$, will read as follows:

$$
\mid \begin{array}{ll}
k u^{\prime \prime}+f=0, & \text { in }] 0,1[, \\
u(0)=u_{0}^{\mathrm{p}}, \quad u(1)=u_{1}^{\mathrm{p}}, & \\
T_{0} v^{\prime \prime}+g=0, & \text { in }] 0,1[ \\
v(0)=v_{0}^{\mathrm{p},}, \quad v(1)=v_{1}^{\mathrm{p}}, &
\end{array}
$$

where $u_{0}^{\mathrm{p}} \mathbf{e}_{x}+v_{0}^{\mathrm{p}} \mathbf{e}_{y}$ and $u_{1}^{\mathrm{p}} \mathbf{e}_{x}+v_{1}^{\mathrm{p}} \mathbf{e}_{y}$ are the prescribed displacements at both ends $x=0$ and $x=1$.

A fixed rigid obstacle is also considered and described by the function:

$$
y=\psi(x)
$$

The reaction force possibly exerted by this obstacle on the string will be written

$$
r \mathbf{e}_{x}+s \mathbf{e}_{y}
$$

In the above expression, $r$ and $s$ are, respectively, the tangential and normal components of the reaction force with respect to a reference configuration. It should be underlined here that, in the linearized framework that has been adopted here, $r$ and $s$ cannot be distinguished in this approximation from the tangential and normal components of the reaction force with respect to the deformed configuration, since the difference is of higher order. 
Assuming that the contact between the string and the obstacle obeys the dry friction Coulomb law with a friction coefficient denoted by $\mu$, one can read the equations that govern the quasi-static evolution of the elastic string above the obstacle formally as follows:

$$
\mid \begin{array}{ll}
k u^{\prime \prime}+f+r=0, & \text { in }] 0,1\left[\times\left[t_{0}, T\right],\right. \\
r(\hat{u}-\dot{u})+\mu s(|\hat{u}|-|\dot{u}|) \geqq 0, \quad \forall \hat{u} \in \mathbb{R}, & \text { in }] 0,1\left[\times\left[t_{0}, T\right],\right. \\
u(0)=u_{0}^{\mathrm{p}}, \quad u(1)=u_{1}^{\mathrm{p}}, & \text { on }\left[t_{0}, T\right], \\
& \text { in }] 0,1[, \\
T_{0} v^{\prime \prime}+g+s=0, & \text { in }] 0,1\left[\times\left[t_{0}, T\right],\right. \\
v-\psi \geqq 0, \quad s \geqq 0, \quad s(v-\psi) \equiv 0, & \text { on }\left[t_{0}, T\right] .
\end{array}
$$

It can be easily checked that the pointwise weak formulation of the Coulomb law used here is equivalent to the usual pointwise formulation. It is worth noting that the equations that govern the transverse component $v$ of the displacement are not coupled with the ones that govern the tangential component.

By changing the value $\mu$ of the friction coefficient, one can always suppose $T_{0}=k=1$. This choice will be made systematically in what follows.

\section{Analysis of the "normal problem" for the string}

The problem that governs the transverse component $v$ of the displacement will be solved first. This problem is the same as that arising in the more usual frictionless situation. At every instant, the problem is classically governed by a variational inequality, which is solved using standard tools (see for example [8]). The purpose of the following theorem is to express how the regularity of the dependence of the data on time can be transferred to the solution, in order to obtain some information on the regularity of the normal component $s(t)$ of the reaction force as it will be used as input data in the analysis of the "tangential problem".

Theorem 1. Let us assume that $\psi \in H^{1}(0,1 ; \mathbb{R}), g:\left[t_{0}, T\right] \rightarrow H^{-1}$ and that the functions $v_{0}^{\mathrm{p}}, v_{1}^{\mathrm{p}}:\left[t_{0}, T\right] \rightarrow \mathbb{R}$ satisfy the strong compatibility condition,

$$
\inf _{t \in\left[t_{0}, T\right]} v_{0}^{\mathrm{p}}(t)>\psi(0), \inf _{t \in\left[t_{0}, T\right]} v_{1}^{\mathrm{p}}(t)>\psi(1),
$$

setting the following:

$$
\begin{gathered}
K(t)=\left\{\varphi \in H^{1}(0,1 ; \mathbb{R}) \mid \varphi(0)=v_{0}^{\mathrm{p}}(t), \quad \varphi(1)=v_{1}^{\mathrm{p}}(t),\right. \\
\forall x \in] 0,1[, \quad \varphi(x) \geqq \psi(x)\},
\end{gathered}
$$

then there exists a unique function $v:\left[t_{0}, T\right] \rightarrow H^{1}(0,1 ; \mathbb{R})$ such that

- $\forall t \in\left[t_{0}, T\right], \quad v(t) \in K(t)$,

- $\forall t \in\left[t_{0}, T\right], \quad \forall \varphi \in K(t), \quad \int_{0}^{1} v^{\prime}\left(\varphi^{\prime}-v^{\prime}\right) \geqq\langle g, \varphi-v\rangle_{H^{-1}, H_{0}^{1}}$. 
Moreover, if $v_{0}^{\mathrm{p}}, v_{1}^{\mathrm{p}}:\left[t_{0}, T\right] \rightarrow \mathbb{R}$ and $g:\left[t_{0}, T\right] \rightarrow H^{-1}$ are regulated (with bounded variation, absolutely continuous, and Lipschitz-continuous, respectively), then the same is true of the function $v:\left[t_{0}, T\right] \rightarrow H^{1}$, and therefore of the function $-v^{\prime \prime}-g \stackrel{\text { def }}{=} s:\left[t_{0}, T\right] \rightarrow H^{-1}$.

Also, for every $t \in\left[t_{0}, T\right], s(t)$ is a positive measure with support contained in $[\alpha, \beta] \subset] 0,1[(\alpha, \beta$ independent of $t)$, and its total mass is a bounded function of $t$.

Proof. Step 1. Existence of $v(t)$.

For every $t \in\left[t_{0}, T\right]$, we take $w(\cdot, t) \in H^{1}(0,1 ; \mathbb{R})$ to denote the solution of the linear problem:

$$
\left.\mid \begin{array}{l}
w^{\prime \prime}+g=0, \\
w(0)=v_{0}^{\mathrm{p}}, \quad
\end{array} \quad \text { in }\right] 0,1[,
$$

It can be readily checked that if $v_{0}^{\mathrm{p}}, v_{1}^{\mathrm{p}}:\left[t_{0}, T\right] \rightarrow \mathbb{R}$ and $g:\left[t_{0}, T\right] \rightarrow H^{-1}$ are regulated (with bounded variation, absolutely continuous, and Lipschitz-continuous, respectively), then the same will be true of the function $w:\left[t_{0}, T\right] \rightarrow H^{1}$.

Let us then proceed by changing the unknown function

$$
\bar{v}(x, t)=v(x, t)-w(x, t) .
$$

Setting

$$
\bar{K}(t)=\left\{\varphi \in H_{0}^{1} \mid \forall x \in\right] 0,1[, \quad \varphi(x) \geqq \psi(x)-w(x, t)\},
$$

one must now prove the existence of a unique function $\bar{v}:\left[t_{0}, T\right] \rightarrow H_{0}^{1}(0,1 ; \mathbb{R})$, having the required regularity in time and satisfying

$$
\begin{aligned}
& \text { - } \forall t \in\left[t_{0}, T\right], \quad \bar{v}(t) \in \bar{K}(t), \\
& \text { - } \forall t \in\left[t_{0}, T\right], \quad \forall \varphi \in \bar{K}(t), \quad \int_{0}^{1} \bar{v}^{\prime}\left(\varphi^{\prime}-\bar{v}^{\prime}\right) \geqq 0 .
\end{aligned}
$$

For every $t \in\left[t_{0}, T\right]$, the use of the Lions-Stampacchia theorem [8] associated with the Poincaré inequality gives a unique $\bar{v}(t) \in \bar{K}(t)$.

Step 2. Properties of the function $s:\left[t_{0}, T\right] \rightarrow H^{-1}$.

It is deduced from the variational inequality satisfied by $\bar{v}(t)$ that at every $t$, the distribution $s(t)=-\bar{v}^{\prime \prime}(t)$ is non-negative (that is, it takes a non-negative value at every $C^{\infty}$ compactly supported non-negative test function). This classically entails that the distribution $s(t)$ is actually a measure.

Since $w$ is a regulated function on $\left[t_{0}, T\right]$ into $H^{1} \subset C^{0}$, given the compactness of the sets $\{0\} \times\left[t_{0}, T\right],\{1\} \times\left[t_{0}, T\right]$ and the conditions (4), one can find $\left.\alpha, \beta \in\right] 0,1[$ such that

$$
\begin{aligned}
& \forall x \in[0, \alpha], \quad \forall t \in\left[t_{0}, T\right], \quad \psi(x)-w(x, t)<0, \\
& \forall x \in[\beta, 1], \quad \forall t \in\left[t_{0}, T\right], \quad \psi(x)-w(x, t)<0 .
\end{aligned}
$$

The support of the measure $s(t)$ is therefore contained in $[\alpha, \beta]$. 
It now remains necessary only to prove that the total mass of this measure is bounded with respect to $t$. Take $s=-\bar{v}^{\prime \prime}$ to denote the measure $s(t)$ at an arbitrarily fixed $t$. For any compact subset $K \in] 0,1[$, there exists a non-negative function $\xi \in C_{0}^{\infty}(] 0,1[)$, which is identically 1 in $K$. For this function

$$
s(K) \leqq \int \xi \mathrm{d} s \leqq\left\|\xi^{\prime}\right\|_{L^{2}}\left\|\bar{v}^{\prime}\right\|_{L^{2}} .
$$

Since

$$
\int_{0}^{1}\left(\bar{v}^{\prime}\right)^{2}=\int_{[0,1]}(\psi-w) \mathrm{d} s \leqq\left\|\langle\psi-w\rangle^{+}\right\|_{L^{\infty}} s\left(\operatorname{supp}\langle\psi-w\rangle^{+}\right),
$$

where $\langle x\rangle^{+}=\max \{x, 0\}$, adopting $K_{1}=\operatorname{supp}\langle\psi-w\rangle^{+}$yields

$$
\int_{0}^{1}\left(\bar{v}^{\prime}\right)^{2} \leqq\left\|\langle\psi-w\rangle^{+}\right\|_{L^{\infty}}\left\|\xi_{1}^{\prime}\right\|_{L^{2}}\left\|\bar{v}^{\prime}\right\|_{L^{2}},
$$

that is

$$
\left\|\bar{v}^{\prime}\right\|_{L^{2}} \leqq\left\|\langle\psi-w\rangle^{+}\right\|_{L^{\infty}}\left\|\xi_{1}^{\prime}\right\|_{L^{2}} .
$$

It then suffices to take $K_{2}=[\alpha, \beta]$ to obtain the required estimate of the total mass of the non-negative measure $s$,

$$
s(] 0,1[)=s([\alpha, \beta]) \leqq\left\|\xi_{1}^{\prime}\right\|_{L^{2}}\left\|\xi_{2}^{\prime}\right\|_{L^{2}}\left\|\langle\psi-w\rangle^{+}\right\|_{L^{\infty}},
$$

since

$$
\|w(t)\|_{L^{\infty}} \leqq C\left\{\left|v_{0}^{\mathrm{p}}(t)\right|+\left|v_{1}^{\mathrm{p}}(t)\right|+\|g(t)\|_{H^{-1}}\right\},
$$

and since any regulated function is bounded.

Step 3. Regularity of the function $\bar{v}:\left[t_{0}, T\right] \rightarrow H_{0}^{1}(0,1 ; \mathbb{R})$.

The claimed regularity of the dependence of the solution on $t$ will be ensured if there exists a constant $C$ which is independent of $t_{1}, t_{2} \in\left[t_{0}, T\right]$, and such that

$$
\left\|\bar{v}\left(t_{2}\right)-\bar{v}\left(t_{1}\right)\right\|_{H_{0}^{1}} \leqq C\left\|w\left(t_{2}\right)-w\left(t_{1}\right)\right\|_{H^{1}} .
$$

Taking arbitrary $t_{1}, t_{2} \in\left[t_{0}, T\right]$ and recalling (5), we set the following:

$$
\begin{aligned}
\bar{\psi}_{i}(\lambda \alpha) & =\lambda\left[\psi(\alpha)-w\left(\alpha, t_{i}\right)\right] \\
\bar{\psi}_{i}(\lambda \alpha+(1-\lambda) \beta) & =\psi(\lambda \alpha+(1-\lambda) \beta)-w\left(\lambda \alpha+(1-\lambda) \beta, t_{i}\right), \\
\bar{\psi}_{i}(\lambda \beta+(1-\lambda)) & =\lambda\left[\psi(\beta)-w\left(\beta, t_{i}\right)\right],
\end{aligned}
$$

for all $\lambda \in[0,1]$ and $i \in\{1,2\}$. The functions $\bar{\psi}_{i}$ defined in this way belong to $H_{0}^{1}$ and satisfy

$$
\left\|\bar{\psi}_{2}^{\prime}-\bar{\psi}_{1}^{\prime}\right\|_{L^{2}} \leqq C\left\|w\left(t_{2}\right)-w\left(t_{1}\right)\right\|_{H^{1}},
$$

where $C$ is a real constant which is independent of $t_{1}, t_{2}$. Moreover, the functions $\bar{\psi}_{i} \in H_{0}^{1}$ differ from $\psi(\cdot)-w\left(\cdot, t_{i}\right)$ only at those $x$ where $\psi(x)-w\left(x, t_{i}\right)<0$. 
Also, the two functions $\bar{v}\left(t_{i}\right)$ are concave, since their second derivatives are nonpositive measures. As they vanish at both ends, these functions are non-negative. Therefore, the function $\bar{v}\left(t_{i}\right)$, which solves the obstacle problem associated with $\psi-w\left(t_{i}\right)$, is also the solution of the obstacle problem associated with $\bar{\psi}_{i}$. From the variational inequalities satisfied by $\bar{v}\left(t_{1}\right)$ and $\bar{v}\left(t_{2}\right)$, respectively, one deduces the following:

$$
\begin{aligned}
& \int_{0}^{1} \bar{v}^{\prime}\left(t_{1}\right)\left[\bar{v}^{\prime}\left(t_{2}\right)-\bar{\psi}_{2}^{\prime}+\bar{\psi}_{1}^{\prime}-\bar{v}^{\prime}\left(t_{1}\right)\right] \geqq 0, \\
& \int_{0}^{1} \bar{v}^{\prime}\left(t_{2}\right)\left[\bar{v}^{\prime}\left(t_{1}\right)-\bar{\psi}_{1}^{\prime}+\bar{\psi}_{2}^{\prime}-\bar{v}^{\prime}\left(t_{2}\right)\right] \geqq 0 .
\end{aligned}
$$

Taking the sum of these two inequalities, we obtain

$$
\int_{0}^{1}\left[\bar{v}^{\prime}\left(t_{2}\right)-\bar{v}^{\prime}\left(t_{1}\right)\right]^{2} \leqq \int_{0}^{1}\left[\bar{v}^{\prime}\left(t_{2}\right)-\bar{v}^{\prime}\left(t_{1}\right)\right]\left[\bar{\psi}_{2}^{\prime}-\bar{\psi}_{1}^{\prime}\right],
$$

and, therefore, reach the desired conclusion (6) by the Cauchy-Schwarz inequality.

\section{Analysis of the "tangential problem"}

\subsection{Structure of the evolution problem}

Once the transverse problem has been solved, the function $s:\left[t_{0}, T\right] \rightarrow \mathcal{M}$ becomes part of the input data in the study on the tangential problem. We now examine the structure of the corresponding evolution problem.

After the unknown $r$ has been eliminated, the problem now consists of finding $u:\left[t_{0}, T\right] \rightarrow H^{1}$ such that

- $u(x, t=0)=u_{0}(x)$,

- $u(x=0, t)=u_{0}^{\mathrm{p}}(t), \quad u(x=1, t)=u_{1}^{\mathrm{p}}(t)$,

- $\forall \varphi \in\{\dot{u}\}+H_{0}^{1}, \quad\left\langle u^{\prime \prime}+f, \varphi-\dot{u}\right\rangle_{H^{-1}, H_{0}^{1}} \leqq\langle\mu s,|\varphi|-|\dot{u}|\rangle_{H^{-1}, H_{0}^{1}}$.

For $\varphi \in H^{1}(0,1 ; \mathbb{R})$, one can set

$$
\bar{\varphi}(x)=\varphi(x)-\varphi(0)-x(\varphi(1)-\varphi(0)) \in H_{0}^{1} .
$$

The isomorphism

$$
\left\{\begin{aligned}
H^{1} & \rightarrow H_{0}^{1} \times \mathbb{R} \times \mathbb{R} \\
\varphi & \mapsto(\bar{\varphi}, \varphi(0), \varphi(1))
\end{aligned}\right.
$$

together with the Poincare inequality can then be used to endow $H^{1}$ with the scalar product defined by

$$
(\varphi \mid \psi)_{H^{1}}=\int_{0}^{1} \bar{\varphi}^{\prime}(x) \bar{\psi}^{\prime}(x) \mathrm{dx}+\varphi(0) \psi(0)+\varphi(1) \psi(1) .
$$


Let us consider the function $\Phi: H^{1} \rightarrow \mathbb{R}$ defined by

$$
\Phi(\varphi)=\langle\mu s,|\varphi|\rangle_{H^{-1}, H_{0}^{1}}-\langle f, \bar{\varphi}\rangle_{H^{-1}, H_{0}^{1}}-u_{0}^{\mathrm{p}} \varphi(0)-u_{1}^{\mathrm{p}} \varphi(1) .
$$

This definition is meaningful since it was noted in the proof of Theorem 1 that supp $s \subset[\alpha, \beta] \subset] 0,1\left[\right.$. The function $\Phi$ is clearly convex and continuous on $H^{1}$. With these notations, one can rewrite the evolution inequality as follows:

$$
\begin{aligned}
\forall \varphi \in & H^{1} \\
& \left\langle u^{\prime \prime}, \bar{\varphi}-\dot{\bar{u}}\right\rangle_{H^{-1}, H_{0}^{1}}-u(0)(\varphi(0)-\dot{u}(0))-u(1)(\varphi(1)-\dot{u}(1)) \\
\leqq & \Phi(\varphi)-\Phi(\dot{u}),
\end{aligned}
$$

that is, since $u^{\prime \prime}=\bar{u}^{\prime \prime}$ :

$$
\begin{aligned}
\forall \varphi \in & H^{1} \\
& -\int_{0}^{1} \bar{u}^{\prime}\left(\bar{\varphi}^{\prime}-\dot{\bar{u}}^{\prime}\right)-u(0)(\varphi(0)-\dot{u}(0))-u(1)(\varphi(1)-\dot{u}(1)) \\
\leqq & \Phi(\varphi)-\Phi(\dot{u}),
\end{aligned}
$$

which, in terms of the subdifferential of the function $\Phi$, simply amounts to

$$
-u(t) \in \partial \Phi[\dot{u}(t)]
$$

where the subdifferential is understood in the sense of the scalar product (7). Since $\Phi$ is positively homogeneous of degree 1 , the conjugate function $\Phi^{*}$ is the indicatrix (in the sense of convex analysis) function of some closed convex set $-\mathcal{C}(t)$. It can then be easily calculated that

$$
\begin{aligned}
& \mathcal{C}(t)=\left\{u \in H^{1} \mid \forall \varphi \in H^{1}, \quad \int_{0}^{1} \bar{u}^{\prime} \bar{\varphi}^{\prime}+u(0) \varphi(0)+u(1) \varphi(1)+\Phi(\varphi) \geqq 0\right\}, \\
&=\left\{u \in H^{1} \mid u(x=0)=u_{0}^{\mathrm{p}}, \quad u(x=1)=u_{1}^{\mathrm{p}},\right. \\
&\text { and } \left.\forall \varphi \in H_{0}^{1}, \quad\left\langle u^{\prime \prime}+f, \varphi\right\rangle_{H^{-1}, H_{0}^{1}} \leqq\langle\mu s,|\varphi|\rangle_{H^{-1}, H_{0}^{1}}\right\},
\end{aligned}
$$

and the problem to be solved is equivalent to that of finding $u:\left[t_{0}, T\right] \rightarrow H^{1}$ such that

- $u\left(t_{0}\right)=u_{0}$,

- $-\dot{u}(t) \in \partial I_{\mathcal{C}(t)}[u(t)]$, for a.a. $t \in\left[t_{0}, T\right]$,

where the subdifferential should be understood with respect to the scalar product (7). The tangential problem, therefore, obeys a sweeping process (see appendix B) in the Hilbert space $H^{1}$. 


\subsection{Existence and uniqueness of strong solutions}

In this section, it is established that the sweeping process that governs the tangential problem can be solved, in some restrictive circumstances, using the results obtained by Moreau (cf. [10] or Appendix B).

Theorem 2. Let $f, s:\left[t_{0}, T\right] \rightarrow H^{-1}$, and $u_{0}^{\mathrm{p}}, u_{1}^{\mathrm{p}}:\left[t_{0}, T\right] \rightarrow \mathbb{R}$. Let us assume that for every $t \in\left[t_{0}, T\right], s(t)$ is a non-negative measure with support contained in some fixed compact interval $[\alpha, \beta] \subset] 0,1[$, and let us consider the set-valued mapping defined by

$$
\begin{aligned}
& \mathcal{C}(t)=\left\{u \in H^{1} \mid u(x=0)=u_{0}^{\mathrm{p}}, \quad u(x=1)=u_{1}^{\mathrm{p}},\right. \\
& \left.\quad \text { and } \forall \varphi \in H_{0}^{1}, \quad\left\langle u^{\prime \prime}+f, \varphi\right\rangle_{H^{-1}, H_{0}^{1}} \leqq\langle\mu s,|\varphi|\rangle_{H^{-1}, H_{0}^{1}}\right\},
\end{aligned}
$$

Some initial condition $u_{0} \in \mathcal{C}(0)$ is also given.

If the functions $u_{0}^{\mathrm{p}}, u_{1}^{\mathrm{p}}:\left[t_{0}, T\right] \rightarrow \mathbb{R}, f:\left[t_{0}, T\right] \rightarrow H^{-1}, s:\left[t_{0}, T\right] \rightarrow \mathcal{M}$ have bounded variation and are right continuous at every $t \in\left[t_{0}, T[\right.$, then the set-valued mapping $\mathcal{C}(t)$ has bounded retraction, and there exists a unique weak solution $u \in B V\left(\left[t_{0}, T\right] ; H^{1}\right)$ of the sweeping process based on $\mathcal{C}(t)$ which agrees with the initial condition $u_{0}$. This weak solution is also the unique solution in the sense of "differential measures", which is right-continuous at every $t \in\left[t_{0}, T[\right.$ (see Appendix B).

If, in addition, the functions $u_{0}^{\mathrm{p}}, u_{1}^{\mathrm{p}}:\left[t_{0}, T\right] \rightarrow \mathbb{R}, f:\left[t_{0}, T\right] \rightarrow H^{-1}$, $s:\left[t_{0}, T\right] \rightarrow \mathcal{M}$ are absolutely continuous (respectively Lipschitz-continuous), then the set-valued mapping $\mathcal{C}(t)$ has absolutely continuous (respectively Lipschitz-continuous) retraction, the solution $u:\left[t_{0}, T\right] \rightarrow H^{1}$ is absolutely continuous (respectively Lipschitz-continuous) and is the unique strong solution of the sweeping process in the sense that

$$
\begin{aligned}
& \text { - } u\left(t_{0}\right)=u_{0}, \\
& \text { - }-\dot{u}(t) \in \partial I_{\mathcal{C}(t)}[u(t)], \quad \text { for a.a. } t \in\left[t_{0}, T\right] .
\end{aligned}
$$

Proof. Taking $e(\cdot, \cdot)$ to denote the "excess" (see Appendix A) associated with the scalar product (7) on $H^{1}$, in order to prove all the claims about the retraction of $\mathcal{C}(t)$, one can prove that at all $t_{1} \leqq t_{2} \in\left[t_{0}, T\right]$

$$
\begin{aligned}
e\left(\mathcal{C}\left(t_{1}\right), \mathcal{C}\left(t_{2}\right)\right) \leqq & C\left\{\left|u_{0}^{\mathrm{p}}\left(t_{2}\right)-u_{0}^{\mathrm{p}}\left(t_{1}\right)\right|+\left|u_{1}^{\mathrm{p}}\left(t_{2}\right)-u_{1}^{\mathrm{p}}\left(t_{1}\right)\right|\right. \\
& \left.+\left\|f\left(t_{2}\right)-f\left(t_{1}\right)\right\|_{H^{-1}}+\left\|\mu s\left(t_{2}\right)-\mu s\left(t_{1}\right)\right\|_{\mathcal{M}}\right\},
\end{aligned}
$$

for some real constant independent of $t_{1}, t_{2}$. We take $w_{i}(i=1,2)$ to denote the unique solution in $H^{1}$ of the linear problem

$$
\mid \begin{aligned}
& w_{i}^{\prime \prime}+f\left(t_{i}\right)=0, \\
& w_{i}(0)=u_{0}^{\mathrm{p}}\left(t_{i}\right), \quad w_{i}(1)=u_{1}^{\mathrm{p}}\left(t_{i}\right),
\end{aligned}
$$

$s_{i}=\mu s\left(t_{i}\right)$, and 


$$
\overline{\mathcal{C}}_{i}=\left\{u \in H_{0}^{1} \mid \forall \varphi \in H_{0}^{1}, \quad\left\langle u^{\prime \prime}, \varphi\right\rangle_{H^{-1}, H_{0}^{1}} \leqq\left\langle s_{i},|\varphi|\right\rangle_{H^{-1}, H_{0}^{1}}\right\},
$$

so that, according to these notations

$$
\mathcal{C}\left(t_{i}\right)=\left\{w_{i}\right\}+\overline{\mathcal{C}}_{i} .
$$

Since the "excess" obeys a triangle inequality (see Proposition 2 in Appendix A):

$$
e\left(\mathcal{C}\left(t_{1}\right), \mathcal{C}\left(t_{2}\right)\right) \leqq\left\|w_{2}-w_{1}\right\|_{H^{1}}+e\left(\overline{\mathcal{C}}_{1}, \overline{\mathcal{C}}_{2}\right) .
$$

The desired inequality will, therefore, be proved provided by

$$
e\left(\overline{\mathcal{C}}_{1}, \overline{\mathcal{C}}_{2}\right) \leqq C\left\|s_{2}-s_{1}\right\|_{\mathcal{M}},
$$

that is, arbitrarily choosing some $\bar{u}_{1} \in \overline{\mathcal{C}}_{1}$ :

$$
d\left(\bar{u}_{1}, \overline{\mathcal{C}}_{2}\right) \leqq C\left\|s_{2}-s_{1}\right\|_{\mathcal{M}},
$$

or

$$
\inf _{\bar{v} \in \overline{\mathcal{C}}_{2}}\left\|\bar{u}_{1}^{\prime}-\bar{v}^{\prime}\right\|_{L^{2}} \leqq C\left\|s_{2}-s_{1}\right\|_{\mathcal{M}}
$$

Since $\bar{u}_{1} \in \overline{\mathcal{C}}_{1}, \bar{u}_{1}^{\prime \prime}$ is a measure with support contained in $[\alpha, \beta]$, and we take $\bar{v}_{0}$ to denote the unique function in $H_{0}^{1}$ such that

$$
\bar{v}_{0}^{\prime \prime}=\inf \left\{\sup \left\{\bar{u}_{1}^{\prime \prime}, 0\right\}, s_{2}\right\}+\sup \left\{\inf \left\{\bar{u}_{1}^{\prime \prime}, 0\right\},-s_{2}\right\},
$$

where the "inf" and "sup" should be understood with respect to the partial order in the space of measures. From

$$
-s_{2} \leqq \bar{v}_{0}^{\prime \prime} \leqq s_{2},
$$

we get $\bar{v}_{0} \in \overline{\mathcal{C}}_{2}$ and

$$
-\left|s_{2}-s_{1}\right| \leqq \bar{v}_{0}^{\prime \prime}-\bar{u}_{1}^{\prime \prime} \leqq\left|s_{2}-s_{1}\right|,
$$

which yields

$$
\left\|\bar{v}_{0}^{\prime \prime}-\bar{u}_{1}^{\prime \prime}\right\|_{\mathcal{M}}=\left\|\left|\bar{v}_{0}^{\prime \prime}-\bar{u}_{1}^{\prime \prime}\right|\right\|_{\mathcal{M}} \leqq\left\|\left|s_{2}-s_{1}\right|\right\|_{\mathcal{M}}=\left\|s_{2}-s_{1}\right\|_{\mathcal{M}} .
$$

Since the imbedding of $\mathcal{M}$ in $H^{-1}$ is continuous (in dimension one),

$$
\left\|\bar{u}_{1}^{\prime}-\bar{v}_{0}^{\prime}\right\|_{L^{2}} \leqq C\left\|s_{2}-s_{1}\right\|_{\mathcal{M}},
$$

for a constant $C$ which is independent of $\bar{v}_{0}$ and $\bar{u}_{1}$. The desired conclusion (8) has now been reached.

Theorem 2 is now a straightforward consequence of Moreau's results (Theorems 8 and 10) as regards the solvability of sweeping processes based on set-valued mappings with bounded retraction. 
(1)

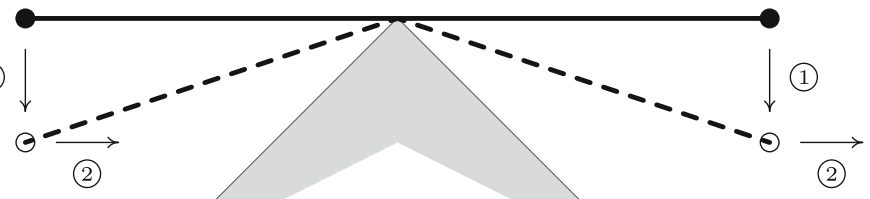

Fig. 1. Elastic string in frictional contact with a wedge-shaped obstacle

\subsection{An example of an explicit solution}

Let us consider the case of the evolution of a string above a fixed rigid wedgeshaped obstacle.

At instant $t=0$, the middle of the string undergoes grazing contact with the top of the obstacle. Between instants $t=0$ and $t=1$, a "vertical" displacement of amplitude $y=-1 / 4$ is imposed on both ends of the string. Then, between instants $t=1$ and $t=2$, a right "horizontal" displacement of the extremities of the string is prescribed at a constant speed (see Fig. 1).

More specifically, this amounts to studying the quasi-static evolution problem for the string associated with the data $\psi(x)=-|x-1 / 2|$, and

$$
\begin{aligned}
& u_{0}^{\mathrm{p}}(t)=0, \quad v_{0}^{\mathrm{p}}(t)=-\frac{t}{4}, \\
& u_{1}^{\mathrm{p}}(t)=0, \quad v_{1}^{\mathrm{p}}(t)=-\frac{t}{4}, \\
& u_{0}^{\mathrm{p}}(t)=\frac{t-1}{4}, \quad v_{0}^{\mathrm{p}}(t)=-\frac{1}{4}, \\
& u_{1}^{\mathrm{p}}(t)=\frac{t-1}{4}, \quad v_{1}^{\mathrm{p}}(t)=-\frac{1}{4}, \\
& \text { for } 0 \leqq t \leqq 1 \text {, } \\
& \text { for } 1 \leqq t \leqq 2 \text {, }
\end{aligned}
$$

It is easily checked that the unique solution of this evolution problem is given by

$$
\begin{aligned}
v(x, t) & =-\frac{t}{2}\left|x-\frac{1}{2}\right|, & u(x, t) & =0, \\
s & =t \delta_{x=1 / 2}, & r & =0,
\end{aligned}
$$

at $0 \leqq t \leqq 1$,

$$
\begin{aligned}
v(x, t) & =-\frac{1}{2}\left|x-\frac{1}{2}\right|, & u(x, t) & =\frac{t-1}{2}\left|x-\frac{1}{2}\right|, \\
s & =\delta_{x=1 / 2}, & r & =(1-t) \delta_{x=1 / 2},
\end{aligned}
$$

at $1 \leqq t \leqq \min (2,1+\mu)$, and in the case $\mu<1$ :

$$
\begin{aligned}
v(x, t) & =-\frac{1}{2}\left|x-\frac{1}{2}\right|, & u(x, t) & =\frac{1}{4}(t-1-\mu)+\frac{\mu}{2}\left|x-\frac{1}{2}\right|, \\
s & =\delta_{x=1 / 2}, & r & =-\mu \delta_{x=1 / 2},
\end{aligned}
$$

at $1+\mu \leqq t \leqq 2$. Thanks to Theorem 2 , the underlying set-valued mapping $\mathcal{C}(t)$ has absolutely continuous (and even Lipschitz-continuous) retraction, and $u$ is a strong solution of the underlying sweeping process. 
Since dry friction is rate-independent, it is natural to attempt to concentrate the episodes of motion prescribed on extremities of the string during the isolated instants $t=0,1$. Setting $u_{0}^{\mathrm{p}}(0)=u_{1}^{\mathrm{p}}(0)=v_{0}^{\mathrm{p}}(0)=v_{1}^{\mathrm{p}}(0)=0$, one considers the following data

$$
\begin{array}{rlrl}
u_{0}^{\mathrm{p}}(t) & =0, & & v_{0}^{\mathrm{p}}(t)=-\frac{1}{4}, \\
u_{1}^{\mathrm{p}}(t)=0, & v_{1}^{\mathrm{p}}(t)=-\frac{1}{4}, & & \text { for } 0<t<1, \\
u_{0}^{\mathrm{p}}(t)=\frac{1}{4}, & v_{0}^{\mathrm{p}}(t)=-\frac{1}{4}, & \\
u_{1}^{\mathrm{p}}(t)=\frac{1}{4}, & v_{1}^{\mathrm{p}}(t)=-\frac{1}{4}, &
\end{array}
$$

The motion of the string is now given by

$$
\begin{aligned}
v(x, t) & =-\frac{1}{2}\left|x-\frac{1}{2}\right|, & u(x, t) & =0, \\
s & =\delta_{x=1 / 2}, & r & =0,
\end{aligned}
$$

at $0<t<1$, and then in the case where $\mu \leqq 1$ by

$$
\begin{aligned}
v(x, t) & =-\frac{1}{2}\left|x-\frac{1}{2}\right|, & u(x, t) & =\frac{1}{2}\left|x-\frac{1}{2}\right|, \\
s & =\delta_{x=1 / 2}, & r & =-\delta_{x=1 / 2},
\end{aligned}
$$

at $1 \leqq t \leqq 2$, and in the case where $\mu \geqq 1$ by

$$
\begin{aligned}
v(x, t) & =-\frac{1}{2}\left|x-\frac{1}{2}\right|, & u(x, t) & =\frac{1}{4}(1-\mu)+\frac{\mu}{2}\left|x-\frac{1}{2}\right|, \\
s & =\delta_{x=1 / 2}, & r & =-\mu \delta_{x=1 / 2},
\end{aligned}
$$

for $1 \leqq t \leqq 2$. In this situation, the moving set $\mathcal{C}(t)$ moves only by translation, but this translation involves two steps. The set-valued mapping $\mathcal{C}(t)$ has right-continuous retraction, the retraction is no longer absolutely continuous, and the function $u$ is a solution of the sweeping process only in the sense of differential measures (see Definition 10).

\subsection{Another example that eludes the theory}

Let us consider the example of a string tightly stretched just above a rigid rectilinear ground. First, a punctual downward force of unit amplitude is applied to the middle of the string. Assuming that the friction coefficient is large (greater than 2), a right displacement of unit amplitude is prescribed on the right extremity of the string. The punctual force then starts to move to the left at a constant speed (see Fig. 2).

More specifically, this amounts to studying the quasi-static evolution problem for the string associated with the following data: $\psi \equiv 0, u_{0}^{\mathrm{p}}=v_{0}^{\mathrm{p}}=v_{1}^{\mathrm{p}} \equiv 0$ and $u_{1}^{\mathrm{p}}$ 


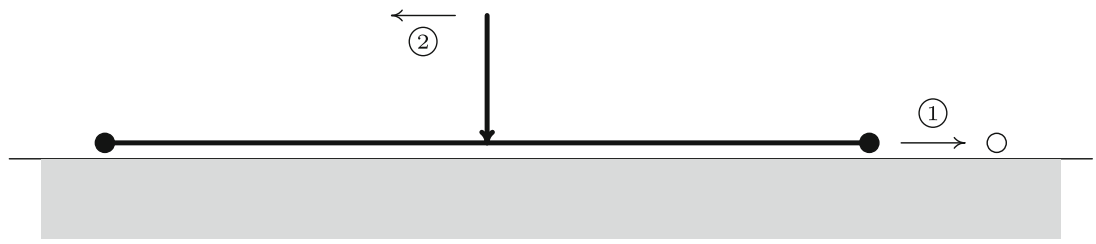

Fig. 2. Frictional contact between an elastic string and a rigid floor

is the function which takes the value 0 at $t=0$ and 1 at every $t>0$. In addition, the body force,

$$
f=\delta_{x=1 / 2-t},
$$

has to be taken into account. The unique solution of the transverse problem is given by $v \equiv 0$, which entails $s \equiv-f$. Since at all $\left.t_{1}<t_{2} \in\right] 0,1[$ :

$$
\begin{aligned}
\left\|\delta_{t_{2}}-\delta_{t_{1}}\right\|_{\mathcal{M}} & =2 \\
\left\|\delta_{t_{2}}-\delta_{t_{1}}\right\|_{H^{-1}} & =\sqrt{t_{2}-t_{1}} \sqrt{1-\left(t_{2}-t_{1}\right)}
\end{aligned}
$$

we have the following regularity for $s$ :

$$
\begin{array}{ll}
s \notin B V([0,1 / 3] ; \mathcal{M}), & s \notin B V\left([0,1 / 3] ; H^{-1}\right), \\
s \notin C^{0}([0,1 / 3] ; \mathcal{M}), & s \in C^{0}\left([0,1 / 3] ; H^{-1}\right) .
\end{array}
$$

This regularity is not sufficiently strong to be able to use Theorem 2 to solve the underlying sweeping process by means of Moreau's results. However, one can consider subdividing $\left[t_{0}, T\right]$, performing the successive projections of the catching-up algorithm, and then attempting to take a limit as the size of the largest interval of the subdivision tends to zero. In the example under consideration, strong convergence in $H^{1}$ occurring uniformly with respect to time is obtained, giving the following weak solution (in line with Definition 9) of the sweeping process:

$$
u(x, t)=\mid \begin{array}{ll}
0, & \text { if } 0 \leqq x \leqq 1 / 2-t \\
\frac{x+t-1 / 2}{t+1 / 2}, & \text { if } 1 / 2-t \leqq x \leqq 1
\end{array}
$$

However, the associated velocity,

$$
\dot{u}(x, t)=\mid \begin{array}{ll}
0, & \text { if } 0 \leqq x<1 / 2-t, \\
\frac{1-x}{(t+1 / 2)^{2}}, & \text { if } 1 / 2-t<x \leqq 1,
\end{array}
$$

shows spatial discontinuity just below the load (see Fig. 3). Therefore, this weak solution does not belong to $B V\left([0,1 / 3] ; H^{1}\right)$, and the underlying set-valued mapping $\mathcal{C}(t)$ cannot have bounded retraction in the Hilbert space $H^{1}$ (see Theorem 8 ). Note, incidentally, that the value of the velocity just below the load is not defined, so that pointwise formulation of the Coulomb law cannot be checked in this problem. 

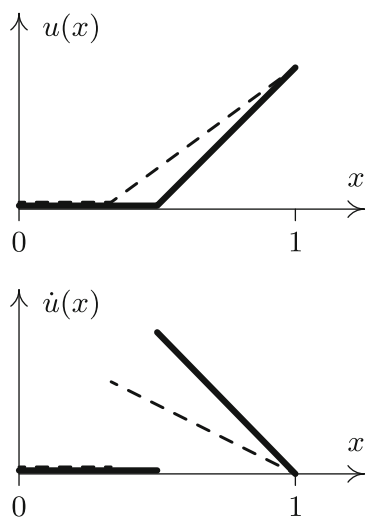

Fig. 3. Longitudinal displacement and velocity at the initial instant as well as at some later instant (dashed lines)

The concept of the weak solution corresponds to subdividing the time interval and introducing the discrete locations of the load associated with the subdivisions. Another way of proceeding would be to "spread out" the load a little bit, by means of a spatial convolution with an approximation of the identity. This is enough to make the underlying set-valued mapping have absolutely continuous (and even Lipschitz-continuous) retraction, and thus to ensure the existence of a strong solution, with a spatially continuous velocity field, in particular. This naturally raises the question as to the existence of a limit, as the regularization tends to identity and the possibility that this limit may coincide with the weak solution, that is, the limit of the solutions of the time-discretized problems.

As an example, let us look at the load, which is homogeneous over the spatial interval $[1 / 2-t-\varepsilon, 1 / 2-t+\varepsilon]$, and of amplitude $1 /(2 \varepsilon)$, where $0<\varepsilon<1 / 6$. It can be easily confirmed that the strong solution of the underlying sweeping process is

$$
u_{\varepsilon}(x, t)=\mid \begin{array}{ll}
0, & \text { if } 0 \leqq x \leqq x_{\varepsilon}(t), \\
\frac{\mu}{4 \varepsilon}\left(x-x_{\varepsilon}(t)\right)^{2}, & \text { if } x_{\varepsilon}(t) \leqq x \leqq \frac{1}{2}-t+\varepsilon, \\
1+\frac{\mu}{2 \varepsilon}\left(\frac{1}{2}-t+\varepsilon-x_{\varepsilon}(t)\right)(x-1), & \text { if } \frac{1}{2}-t+\varepsilon \leqq x \leqq 1 .
\end{array}
$$

where

$$
x_{\varepsilon}(t)=1-\sqrt{\left(\frac{1}{2}+t-\varepsilon\right)^{2}+\frac{4 \varepsilon}{\mu}} \in\left[\frac{1}{2}-t-\varepsilon, \frac{1}{2}-t+\varepsilon\right] .
$$

It is worth noting in this example that $u_{\varepsilon}$ converges towards $u$ as $\varepsilon$ tends to 0 , in a strong sense: strong convergence in $H^{1}$, uniformly with respect to $t \in[0,1 / 3]$.

The solution $u_{\varepsilon}$ provides an explanation of a surprising feature of the solution $u$ of the non-regularized problem. Although the friction coefficient chosen was large enough to prevent any slipping, the elastic energy associated with $u$ decreases 
with respect to time. This fact can be explained as follows. The solution $u_{\varepsilon}$ of the regularized problem always shows some slipping, and it can be checked that the accumulated dissipation (the time integral of the power of the friction force) tends, as $\varepsilon \rightarrow 0$, not towards zero, but towards some finite value. It is, therefore, logical that the weak solution $u$ of the "limit" problem should keep some memory of this dissipation, although showing no slipping itself.

\subsection{Weak solutions}

In this section it is proved, after adopting some fairly general regularity hypotheses about the data involved in the frictional problem, that the set-valued mapping of the underlying sweeping process is Wijsman-regulated. This enables us to state the problem of the possible existence of a weak solution of the frictional contact problem. However, the question of existence of such a weak solution is left open at the moment.

More specifically, we propose to prove that the regularity obtained for the function $s(t)$ by solving the normal problem yields a Wijsman-regulated set-valued mapping $\mathcal{C}(t)$.

Proposition 1. Let $f, s:\left[t_{0}, T\right] \rightarrow H^{-1}$, as well as $u_{0}^{\mathrm{p}}, u_{1}^{\mathrm{p}}:\left[t_{0}, T\right] \rightarrow \mathbb{R}$. Let us assume that for every $t \in\left[t_{0}, T\right], s(t)$ is a non-negative measure having a support which is contained in a fixed compact interval $[\alpha, \beta] \subset] 0,1[$, and a total mass bounded independently of $t$. Let us consider the set-valued mapping defined by

$$
\begin{aligned}
& \mathcal{C}(t)=\left\{u \in H^{1} \mid u(x=0)=u_{0}^{\mathrm{p}}, \quad u(x=1)=u_{1}^{\mathrm{p}},\right. \\
& \left.\quad \text { and } \forall \varphi \in H_{0}^{1}, \quad\left\langle u^{\prime \prime}+f, \varphi\right\rangle_{H^{-1}, H_{0}^{1}} \leqq\langle\mu s,|\varphi|\rangle_{H^{-1}, H_{0}^{1}}\right\},
\end{aligned}
$$

If the functions $f, s:\left[t_{0}, T\right] \rightarrow H^{-1}, u_{0}^{\mathrm{p}}, u_{1}^{\mathrm{p}}:\left[t_{0}, T\right] \rightarrow \mathbb{R}$ are regulated, then the set-valued mapping $\mathcal{C}(t)$ is Wijsman-regulated.

Proof. As in the proof of Theorem 2,w(t) is defined as the unique solution (at fixed $t$ ) of the linear problem

$$
\mid \begin{aligned}
& w^{\prime \prime}+f(t)=0 \\
& w(0)=u_{0}^{\mathrm{p}}(t), \quad w(1)=u_{1}^{\mathrm{p}}(t)
\end{aligned}
$$

and

$$
\overline{\mathcal{C}}(t)=\left\{u \in H_{0}^{1} \mid \forall \varphi \in H_{0}^{1}, \quad \int_{0}^{1} u^{\prime} \varphi^{\prime} \leqq\langle s(t),|\varphi|\rangle_{H^{-1}, H_{0}^{1}}\right\} .
$$

According to these notations

$$
\mathcal{C}(t)=\{w(t)\}+\overline{\mathcal{C}}(t) .
$$


It should be clear that if the three functions $f:\left[t_{0}, T\right] \rightarrow H^{-1}, u_{0}^{\mathrm{p}}, u_{1}^{\mathrm{p}}:\left[t_{0}, T\right] \rightarrow$ $\mathbb{R}$ are regulated, then the same will be true of the function $w:\left[t_{0}, T\right] \rightarrow H^{1}$. Setting

$$
\begin{aligned}
\overline{\mathcal{C}}_{n} & =\left\{u \in H_{0}^{1} \mid \forall \varphi \in H_{0}^{1}, \quad \int_{0}^{1} u^{\prime} \varphi^{\prime} \leqq\left\langle s_{n},|\varphi|\right\rangle_{H^{-1}, H_{0}^{1}}\right\}, \\
\overline{\mathcal{C}} & =\left\{u \in H_{0}^{1} \mid \forall \varphi \in H_{0}^{1}, \quad \int_{0}^{1} u^{\prime} \varphi^{\prime} \leqq\langle s,|\varphi|\rangle_{H^{-1}, H_{0}^{1}}\right\}
\end{aligned}
$$

(where $s_{n}$ and $s$ are non-negative measures with their support in $[\alpha, \beta]$, having a total mass which is bounded independently of $n$ ) and taking into account Theorem 4 , one must now prove that if the sequence $\left(s_{n}\right)$ converges strongly towards $s$ in $H^{-1}$, then $\lim _{n \rightarrow \infty} \overline{\mathcal{C}}_{n}=\overline{\mathcal{C}}$, in the sense of Kuratowski.

Choosing $u \in \lim \sup _{n \rightarrow \infty} \overline{\mathcal{C}}_{n}$ arbitrarily, one finds that there exists a subsequence of $\left(s_{n}\right)$, which is still denoted by $\left(s_{n}\right)$, and a sequence $\left(u_{n}\right)$ in $H_{0}^{1}$ such that $\left(u_{n}^{\prime}\right)$ converges strongly towards $u^{\prime}$ in $L^{2}$ and

$$
\forall \varphi \in H_{0}^{1}, \quad \forall n \in \mathbb{N}, \quad \int_{0}^{1} u_{n}^{\prime} \varphi^{\prime} \leqq\left\langle s_{n},|\varphi|\right\rangle_{H^{-1}, H_{0}^{1}} .
$$

If $n$ tends to infinity, it can be seen that $u \in \overline{\mathcal{C}}$; hence, $\lim _{\sup _{n \rightarrow \infty}} \overline{\mathcal{C}}_{n} \subset \overline{\mathcal{C}}$.

Now let us take arbitrary $u \in \overline{\mathcal{C}}$. Noting that $u^{\prime \prime}$ is a measure with support in $[\alpha, \beta]$, set

$$
\bar{u}_{n}^{\prime \prime}=\inf \left\{\sup \left\{\bar{u}^{\prime \prime}, 0\right\}, s_{n}\right\}+\sup \left\{\inf \left\{\bar{u}^{\prime \prime}, 0\right\},-s_{n}\right\},
$$

where the infimum and supremum should be understood in terms of the partial ordering in the space of measures. As

$$
-s_{n} \leqq \bar{u}_{n}^{\prime \prime} \leqq s_{n}
$$

we obtain $\bar{u}_{n} \in \overline{\mathcal{C}}_{n}$. Now, remember that a sequence $\left(f_{n}\right)$ in the dual space $X^{\prime}$ of a Banach space $X$ converges weakly-star towards $f$ if and only if $\left\|f_{n}\right\|$ is bounded, and if $\left\langle f_{n}, x\right\rangle \rightarrow\langle f, x\rangle$ for every $x$ in a dense subset of $X$ (see [13], theorem 10, p.125). Since the total mass of $s_{n}$ is bounded and since the restrictions of functions in $H_{0}^{1}$ to the interval $[\alpha, \beta]$ are dense in $C^{0}([\alpha, \beta])$, it is deduced that the strong convergence of $s_{n}$ towards $s$ in $H^{-1}$ entails the weak-star convergence of $s_{n}$ towards $s$ in $\mathcal{M}([\alpha, \beta])$. From the definition of $\bar{u}_{n}^{\prime \prime}$ in terms of $u \in \overline{\mathcal{C}}$, then we have the weak-star convergence of $u_{n}^{\prime \prime}$ towards $u^{\prime \prime}$ in $\mathcal{M}([\alpha, \beta])$. First, this entails pointwise convergence almost everywhere of $\bar{u}_{n}^{\prime}$ towards $\bar{u}^{\prime}$, and then, by dominated convergence, strong convergence in $L^{2}$ of $\bar{u}_{n}^{\prime}$ towards $\bar{u}^{\prime}$; hence $u \in \liminf _{n \rightarrow \infty} \overline{\mathcal{C}}_{n}$.

Upon combining all these elements, we obtain

$$
\limsup _{n \rightarrow \infty} \overline{\mathcal{C}}_{n} \subset \overline{\mathcal{C}} \subset \liminf _{n \rightarrow \infty} \overline{\mathcal{C}}_{n}
$$

which is the conclusion we were looking for. 


\section{Replacing the string by a beam}

Let us consider a straight beam which is simply supported at both ends and has as its initial configuration the segment $[0,1] \times\{0\}$. The linearized equations that govern the equilibrium of the beam, which is assumed to be elastic, read as follows:

$$
\mid \begin{array}{ll}
k u^{\prime \prime}+f=0, & \text { in }] 0,1[, \\
u(0)=u_{0}^{\mathrm{p}}, \quad u(1)=u_{1}^{\mathrm{p}}, & \\
l v^{\prime \prime \prime \prime}-g=0, & \text { in }] 0,1[, \\
v(0)=v_{0}^{\mathrm{p}}, \quad v(1)=v_{1}^{\mathrm{p}}, & \\
v^{\prime \prime}(0)=v^{\prime \prime}(1)=0, &
\end{array}
$$

where the traction stiffness $k$ and the flexion stiffness $l$ will equal 1 in what follows by choosing the unit appropriately, and $u_{0}^{\mathrm{p}} \mathbf{e}_{x}+v_{0}^{\mathrm{p}} \mathbf{e}_{y}$ and $u_{1}^{\mathrm{p}} \mathbf{e}_{x}+v_{1}^{\mathrm{p}} \mathbf{e}_{y}$ are the prescribed displacements at extremities $x=0$ and $x=1$, respectively.

The equations governing the quasi-static evolution of the beam above a fixed rigid obstacle of equation $y=\psi(x)$ with Coulomb dry friction of coefficient denoted by $\mu$, can be written as follows:

$$
\mid \begin{array}{ll}
u^{\prime \prime}+f+r=0, & \text { in }] 0,1\left[\times\left[t_{0}, T\right],\right. \\
r(\hat{u}-\dot{u})+\mu s(|\hat{u}|-|\dot{u}|) \geqq 0, \quad \forall \hat{u} \in \mathbb{R}, & \text { in }] 0,1\left[\times\left[t_{0}, T\right],\right. \\
u(0)=u_{0}^{\mathrm{p}}, \quad u(1)=u_{1}^{\mathrm{p}}, & \text { on }\left[t_{0}, T\right], \\
v^{\prime \prime \prime \prime}-g-s=0, & \text { in }] 0,1[, \\
v-\psi \geqq 0, \quad s \geqq 0, \quad s(v-\psi) \equiv 0, & \text { in }] 0,1\left[\times\left[t_{0}, T\right],\right. \\
v(0)=v_{0}^{\mathrm{p}}, \quad v(1)=v_{1}^{\mathrm{p}}, & \text { on }\left[t_{0}, T\right], \\
v^{\prime \prime}(0)=v^{\prime \prime}(1)=0, & \text { on }\left[t_{0}, T\right] .
\end{array}
$$

The equations governing the normal component $v$ of the displacement are still uncoupled with those governing the tangential component.

\subsection{Another example}

It could seem at first sight that the case of the beam brings nothing more to the case of the string, except that the order of the differential operator in the variational inequality that governs the normal displacement is 4 instead of 2 , whereas the problems governing the tangential displacement remains formally the same in both cases.

This is true, but the fact that the operator governing the normal displacement is now of order 4 has some important effects. In particular, one can expect the solutions of the underlying sweeping process be be weak solutions, even when arbitrarily smooth data are available. This can be confirmed by analysing the problem with the geometry shown in Fig. 4. In the initial configuration, the beam undergoes grazing contact with a smooth obstacle. The amplitude of the force is made to increase gradually with time $t$. It can easily be checked that the contact zone in the solution reduces to a single point, provided the amplitude of the force is small enough, and that this punctual contact zone is associated with a point on the obstacle that moves to the left of the figure with time. Consequently, the normal reaction $s$ is a Dirac 


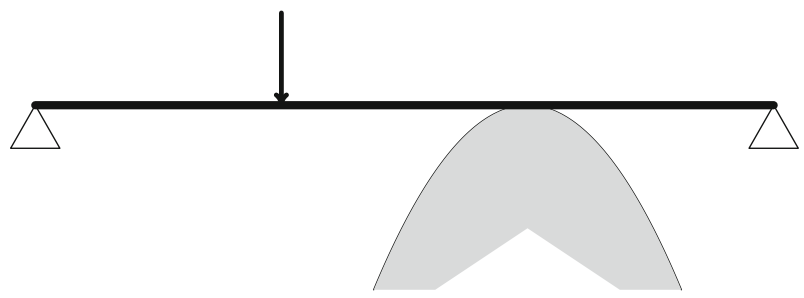

Fig. 4. Frictional contact of a simply supported beam

measure whose support moves with time, as in the example given in Fig. 2. This fact will be true even in cases where the external force is "spread out" a little bit so as to be as smooth as desired. Therefore, one cannot expect to obtain

$$
s \in B V\left(\left[t_{0}, T\right] ; \mathcal{M}\right),
$$

by requiring the data to be smooth. The tangential problem will, therefore, generally have only weak solutions, even with smooth data.

\subsection{About weak solutions}

In this section, the regularity that can be expected to occur with the function $s(t)$, and therefore with the set-valued mapping $\mathcal{C}(t)$, will be analysed in the case of beams, where the variational inequality is associated with the biharmonic operator instead of the harmonic one. It is worth noting that under the same regularity assumptions about the data, the function $s(t)$ shows the same regularity here as in Theorem 1. This is stated in the following theorem, in which combines several regularity results that are known for variational inequalities associated with the biharmonic operator.

Once Theorem 3 has been proved, Proposition 1 ensures that the underlying set-valued mapping $\mathcal{C}(t)$ is Wijsman-regulated, provided the data $f, g:\left[t_{0}, T\right] \rightarrow$ $H^{-1}, u_{0}^{\mathrm{p}}, v_{0}^{\mathrm{p}}, u_{1}^{\mathrm{p}}, v_{1}^{\mathrm{p}}:\left[t_{0}, T\right] \rightarrow \mathbb{R}$ are regulated functions.

Theorem 3. Let us assume that $\psi \in H^{3}(0,1 ; \mathbb{R}), g:\left[t_{0}, T\right] \rightarrow H^{-1}$ and that the functions $v_{0}^{\mathrm{p}}, v_{1}^{\mathrm{p}}:\left[t_{0}, T\right] \rightarrow \mathbb{R}$ satisfy the strong compatibility condition,

$$
\inf _{t \in\left[t_{0}, T\right]} v_{0}^{\mathrm{p}}(t)>\psi(0), \inf _{t \in\left[t_{0}, T\right]} v_{1}^{\mathrm{p}}(t)>\psi(1),
$$

setting:

$$
\begin{gathered}
K(t)=\left\{\hat{v} \in H^{2}(0,1 ; \mathbb{R}) \mid \hat{v}(0)=v_{0}^{\mathrm{p}}(t), \quad \hat{v}(1)=v_{1}^{\mathrm{p}}(t),\right. \\
\forall x \in] 0,1[, \quad \hat{v}(x) \geqq \psi(x)\},
\end{gathered}
$$

then there exists a unique function $v:\left[t_{0}, T\right] \rightarrow H^{2}(0,1 ; \mathbb{R})$ such that

- $\forall t \in\left[t_{0}, T\right], \quad v(t) \in K(t)$,

- $\forall t \in\left[t_{0}, T\right], \quad \forall \hat{v} \in K(t), \quad \int_{0}^{1} v^{\prime \prime}\left(\hat{v}^{\prime \prime}-v^{\prime \prime}\right) \geqq\langle g, \hat{v}-v\rangle_{H^{-1}, H_{0}^{1}}$. 
Moreover, if $v_{0}^{\mathrm{p}}, v_{1}^{\mathrm{p}}:\left[t_{0}, T\right] \rightarrow \mathbb{R}$ and $g:\left[t_{0}, T\right] \rightarrow H^{-1}$ are regulated, then the same will be true of the function $v:\left[t_{0}, T\right] \rightarrow H^{3}$, and, therefore, of the function $v^{\prime \prime \prime \prime}-g \stackrel{\text { def }}{=} s:\left[t_{0}, T\right] \rightarrow H^{-1}$.

Also, for every $t \in\left[t_{0}, T\right], s(t)$ is a non-negative measure with support contained in $[\alpha, \beta] \subset] 0,1[(\alpha, \beta$ are independent of $t)$, whose total mass is a bounded function of $t$.

Proof. This additional regularity $\left(H^{3}\right.$ instead of $\left.H^{2}\right)$ shown by the solutions of the obstacle problem associated with the biharmonic operator is a well-known fact. Here we reproduce the proof by penalization displayed in [8, p. 270] (the reader will find there the bibliographical references on the subject), because it can readily be transposed to higher space dimensions and, in particular, to the case of the plate. To prove that the mapping $v:\left[t_{0}, T\right] \rightarrow H^{3}$ thus defined is regulated, we shall use the fact that a mapping with values in a complete metric space is regulated if and only if it admits a left limit and a right limit at every point. Thus, the problem is made to focus on the stability of the solution to the biharmonic obstacle problem with respect to the data. This stability problem was studied by ADAMs [1], whose results are very similar to those needed here. Our method of proof is on very similar lines to those used in [1].

Step 1. Existence and uniqueness of the function $v:\left[t_{0}, T\right] \rightarrow H^{2}$.

At every $t \in\left[t_{0}, T\right]$, we take $w(\cdot, t) \in H^{2}(0,1 ; \mathbb{R})$ to denote the solution of the linear problem

$$
\mid \begin{array}{ll}
w^{\prime \prime \prime \prime}-g=0, & \text { in }] 0,1[ \\
w(0)=v_{0}^{\mathrm{p}}, \quad w(1)=v_{1}^{\mathrm{p}}, & \\
w^{\prime \prime}(0)=w^{\prime \prime}(1)=0 . &
\end{array}
$$

It should be clear that $w(\cdot, t) \in H^{3}(0,1 ; \mathbb{R})$ and that the linear mapping

$$
\left\{\begin{aligned}
\mathbb{R} \times \mathbb{R} \times H^{-1} & \rightarrow H^{3} \\
\left(v_{0}^{\mathrm{p}}(t), v_{1}^{\mathrm{p}}(t), g(t)\right) & \mapsto w(t)
\end{aligned}\right.
$$

is continuous. In particular, if the data are regulated functions of the variable $t$, then the same will be true of the function $w:\left[t_{0}, T\right] \rightarrow H^{3}$. Next, we proceed with changing the unknown function

$$
\bar{v}(x, t)=v(x, t)-w(x, t),
$$

and set

$$
\bar{K}(t)=\left\{\hat{v} \in H_{0}^{1} \cap H^{2} \mid \forall x \in\right] 0,1[, \quad \hat{v}(x) \geqq \psi(x)-w(t, x)\} .
$$

By the Lions-Stampacchia theorem, there exists a unique $\bar{v}(t) \in \bar{K}(t)$ such that

$$
\forall \hat{v} \in \bar{K}(t), \quad \int_{0}^{1} \bar{v}^{\prime \prime}\left(\hat{v}^{\prime \prime}-\bar{v}^{\prime \prime}\right) \geqq 0,
$$


provided that the bilinear form $(v, w) \rightarrow \int_{0}^{1} v^{\prime \prime} w^{\prime \prime}$ is coercive on the Hilbert space $H_{0}^{1} \cap H^{2}$ equipped with the norm

$$
\|v\|_{H_{0}^{1} \cap H^{2}}=\sqrt{\left\|v^{\prime}\right\|_{L_{2}}^{2}+\left\|v^{\prime \prime}\right\|_{L_{2}}^{2}} .
$$

Take $v \in H_{0}^{1} \cap H^{2} \subset C^{1}$. There exists $\left.x_{0} \in\right] 0,1\left[\right.$ such that $v^{\prime}\left(x_{0}\right)=0$. We obtain

$$
\left[v^{\prime}(x)\right]^{2}=2 \int_{x_{0}}^{x} v^{\prime} v^{\prime \prime} \leqq 2 \sqrt{\int_{0}^{1} v^{\prime 2}} \sqrt{\int_{0}^{1} v^{\prime \prime 2}}
$$

which entails

$$
\sqrt{\int_{0}^{1} v^{\prime 2}} \leqq 2 \sqrt{\int_{0}^{1} v^{\prime \prime 2}}
$$

(this is, in fact, the desired coerciveness); therefore, the existence of a unique $\bar{v}(t) \in \bar{K}(t)$ solving the variational inequality.

It is now proposed to prove that it is always possible to reduce the problem to the case where the obstacle is described by a function which vanishes at the extremities $x=0,1$. The function $\bar{\psi}(x)$ will be constructed as in the proof of Theorem 1 . Since $w:\left[t_{0}, T\right] \rightarrow H^{3}$ is regulated, by the conditions pertaining in (4), one can find $\alpha, \beta \in] 0,1[$ such that

$$
\begin{array}{lll}
\forall x \in[0, \alpha], & \forall t \in\left[t_{0}, T\right], & \psi(x)-w(x, t)<0, \\
\forall x \in[\beta, 1], & \forall t \in\left[t_{0}, T\right], & \psi(x)-w(x, t)<0 .
\end{array}
$$

The function $\bar{\psi}(x, t)$ can then be defined by

$$
\begin{aligned}
\bar{\psi}(\lambda \alpha, t)= & {\left[\lambda^{3}-3 \lambda^{2}+3 \lambda\right][\psi(\alpha)-w(\alpha, t)] } \\
& -\left[\lambda^{3}-3 \lambda^{2}+2 \lambda\right]\left[\psi^{\prime}(\alpha)-\frac{\partial w}{\partial x}(\alpha, t)\right] \alpha \\
& +\left[\lambda^{3}-2 \lambda^{2}+\lambda\right]\left[\psi^{\prime \prime}(\alpha)-\frac{\partial^{2} w}{\partial x^{2}}(\alpha, t)\right] \frac{\alpha^{2}}{2}, \\
\bar{\psi}(\lambda \alpha+(1-\lambda) \beta, t)= & \psi(\lambda \alpha+(1-\lambda) \beta)-w(\lambda \alpha+(1-\lambda) \beta, t), \\
\bar{\psi}(\lambda \beta+(1-\lambda), t)= & {\left[\lambda^{3}-3 \lambda^{2}+3 \lambda\right][\psi(\beta)-w(\beta, t)] } \\
& -\left[\lambda^{3}-3 \lambda^{2}+2 \lambda\right]\left[\psi^{\prime}(\beta)-\frac{\partial w}{\partial x}(\beta, t)\right](1-\beta) \\
& +\left[\lambda^{3}-2 \lambda^{2}+\lambda\right]\left[\psi^{\prime \prime}(\beta)-\frac{\partial^{2} w}{\partial x^{2}}(\beta, t)\right] \frac{(1-\beta)^{2}}{2},
\end{aligned}
$$

for every $\lambda \in[0,1]$. It can be readily checked that $\bar{\psi}(t) \in H_{0}^{1} \cap H^{3}$ and that

$$
\|\bar{\psi}(t)\|_{H^{3}} \leqq C\|\psi-w(t)\|_{H^{3}},
$$


for a real constant $C$ which depends only on $\alpha$ and $\beta$ (and is therefore independent of $t$ and $w(t)$ ). Moreover, $\bar{v}^{\prime \prime}$ is convex and vanishes at extremities $x=0,1$. It is therefore non-positive, and $\bar{v}(t)$ is a concave function of $x$. Hence, it is non-negative. Since the function $\bar{\psi}(\cdot, t)$ differs from $\psi(\cdot)-w(\cdot, t)$ only at those values of $x$ where the latter is negative, this entails that $\bar{v}$, which solves the obstacle problem associated with $\psi-w$, also solves the obstacle problem associated with $\bar{\psi}$.

Step $2 . H^{3}$ regularity of the solution at every instant.

In step 2, an arbitrary $t$ in $\left[t_{0}, T\right]$ is fixed once for all.

Define $\bar{g}=\bar{\psi}^{\prime \prime \prime \prime} \in H^{-1}$ to be able to proceed with changing the unknown function:

$$
\overline{\bar{v}}=\bar{v}-\bar{\psi}
$$

so that, setting

$$
\overline{\bar{K}}=\left\{\hat{v} \in H_{0}^{1} \cap H^{2} \mid \forall x \in\right] 0,1[, \quad \hat{v}(x) \geqq 0\},
$$

one obtains $\overline{\bar{v}} \in \overline{\bar{K}}$ and

$$
\forall \hat{v} \in \overline{\bar{K}}, \quad \int_{0}^{1} \overline{\bar{v}}^{\prime \prime}\left(\hat{v}^{\prime \prime}-\overline{\bar{v}}^{\prime \prime}\right) \geqq\langle\bar{g}, \hat{v}-\overline{\bar{v}}\rangle_{H^{-1}, H_{0}^{1}} .
$$

As in [8, p. 270], for every $\varepsilon>0$, the penalized function $p_{\varepsilon}$ is defined as the unique solution in $H^{2}(0,1 ; \mathbb{R})$ of the linear boundary problem

$$
\mid \begin{aligned}
& \left.p_{\varepsilon}-\varepsilon p_{\varepsilon}^{\prime \prime}=\overline{\bar{v}}, \quad \text { in }\right] 0,1[ \\
& p_{\varepsilon}(0)=p_{\varepsilon}(1)=0
\end{aligned}
$$

It can be readily seen that

- $p_{\varepsilon} \in H^{4}(0,1 ; \mathbb{R})$,

- $p_{\varepsilon}^{\prime \prime}(0)=p_{\varepsilon}^{\prime \prime}(1)=0$.

Moreover, if $p_{\varepsilon}\left(x_{0}\right)=\min _{[0,1]} p_{\varepsilon}$ for some $\left.x_{0} \in\right] 0,1\left[\right.$, then $p_{\varepsilon}^{\prime \prime}\left(x_{0}\right) \geqq 0$; therefore, $p_{\varepsilon}\left(x_{0}\right) \geqq \overline{\bar{v}}\left(x_{0}\right) \geqq 0$. This entails

$$
\forall \varepsilon>0, \quad p_{\varepsilon} \in \overline{\bar{K}}
$$

But, for all $\hat{v} \in \overline{\bar{K}}$,

$$
\int_{0}^{1} \hat{v}^{\prime \prime}\left(\hat{v}^{\prime \prime}-\overline{\bar{v}}^{\prime \prime}\right) \geqq \int_{0}^{1} \overline{\bar{v}}^{\prime \prime}\left(\hat{v}^{\prime \prime}-\overline{\bar{v}}^{\prime \prime}\right) \geqq\langle\bar{g}, \hat{v}-\overline{\bar{v}}\rangle_{H^{-1}, H_{0}^{1}} .
$$

Applying this inequality to the case $\hat{v}=p_{\varepsilon}$, one gets

$$
\int_{0}^{1} p_{\varepsilon}^{\prime \prime \prime \prime} p_{\varepsilon}^{\prime \prime} \geqq\left\langle\bar{g}, p_{\varepsilon}^{\prime \prime}\right\rangle_{H^{-1}, H_{0}^{1}}
$$


But $\bar{g}=-G^{\prime}$ for some $G \in L^{2}$, and one obtains

$$
\int_{0}^{1} p_{\varepsilon}^{\prime \prime \prime \prime} p_{\varepsilon}^{\prime \prime} \geqq \int_{0}^{1} G p_{\varepsilon}^{\prime \prime \prime},
$$

that is,

$$
\int_{0}^{1}\left(p_{\varepsilon}^{\prime \prime \prime}\right)^{2} \leqq-\int_{0}^{1} G p_{\varepsilon}^{\prime \prime \prime}
$$

and as a result:

$$
\left\|p_{\varepsilon}^{\prime \prime \prime}\right\|_{L^{2}} \leqq\|G\|_{L^{2}}=\|\bar{g}\|_{H^{-1}} .
$$

By Poincaré inequality

$$
\left\|p_{\varepsilon}^{\prime \prime}\right\|_{L^{2}} \leqq C\left\|p_{\varepsilon}^{\prime \prime \prime}\right\|_{L^{2}},
$$

for a constant $C$ independent of $\varepsilon$. Recalling $p_{\varepsilon} \in H_{0}^{1} \cap H^{2}$ and inequality (10), one obtains

$$
\forall \varepsilon>0, \quad\left\|p_{\varepsilon}\right\|_{H^{3}} \leqq C\|\bar{g}\|_{H^{-1}},
$$

for a constant $C$ independent of $\varepsilon$, as well as of $\bar{g}$. This inequality yields $\left\|p_{\varepsilon}^{\prime \prime}\right\|_{L^{2}} \leqq$ $C\|\bar{g}\|_{H^{-1}}$, first, and $\left\|p_{\varepsilon}-\overline{\bar{v}}\right\|_{L^{2}} \leqq C \varepsilon\|\bar{g}\|_{H^{-1}}$, then, which shows that $p_{\varepsilon}$ tends towards $\overline{\bar{v}}$ strongly in $L^{2}$, as $\varepsilon$ tends to $0+$. Also, by virtue of (12), there exists a subsequence converging weakly in $H^{3}$. Since weak convergence in $H^{3}$ is, in particular, strong convergence in $L^{2}$, the limit must be $\overline{\bar{v}}$, which therefore belongs to $H^{3}$.

Step 3. Regularity of the dependence of the solution on time.

Since a function with values in a complete metric space is regulated if and only if it admits a left limit and a right limit at every point, it suffices to prove the following stability result:

$$
\lim _{n \rightarrow+\infty}\left\|w-w_{n}\right\|_{H^{3}}=0 \quad \Longrightarrow \quad \lim _{n \rightarrow+\infty}\left\|\bar{v}-\bar{v}_{n}\right\|_{H^{3}}=0
$$

where $\bar{v}$ (respectively $\bar{v}_{n}$ ) is the solution of inequality (9) involving the data $w$ (respectively $w_{n}$ ).

The proof of this stability result is largely inspired by ADAMs' technique [1].

Denote $s_{n}=\bar{v}_{n}^{\prime \prime \prime \prime}$ (respectively, $s=\bar{v}^{\prime \prime \prime \prime}$ ). These distributions are non-negative (that is, they take non-negative values at every $C^{\infty}$ test-function with compact support), and there are, therefore, some measures. A double integration by parts yields

$$
\int_{0}^{1}\left(\bar{v}^{\prime \prime}-\bar{v}_{n}^{\prime \prime}\right)^{2}=\int_{0}^{1}\left(\bar{v}-\bar{v}_{n}\right) \mathrm{d}\left(s-s_{n}\right) \leqq \int_{0}^{1}\left(w_{n}-w\right) \mathrm{d}\left(s-s_{n}\right),
$$

since $\bar{v}_{n}=\psi-w_{n}$ on $\operatorname{supp} s_{n}(\bar{v}=\psi-w$ on $\operatorname{supp} s)$ and $\bar{v}_{n} \geqq \psi-w_{n}$ on $[0,1]$ $(\bar{v} \geqq \psi-w$ on $[0,1])$. This entails

$$
\lim _{n \rightarrow+\infty}\left\|\bar{v}-\bar{v}_{n}\right\|_{H^{2}}=0,
$$


provided the total mass of the non-negative measure $s_{n}=\bar{v}_{n}^{\prime \prime \prime \prime}$ is bounded independently of $n$. To prove this, take $[\alpha, \beta] \subset] 0,1\left[\right.$ such that $\psi-w_{n}<0$ on $] 0,1[\backslash[\alpha, \beta]$. Since $\bar{v}_{n} \geqq 0, \operatorname{supp} s_{n} \subset[\alpha, \beta]$. Moreover, for every compact set $\left.K \in\right] 0,1$ [, one can find a non-negative function $\xi \in C_{0}^{\infty}(] 0,1[)$, which equals 1 identically on $K$. This entails

$$
s_{n}(K) \leqq \int \xi \mathrm{d} s_{n} \leqq\left\|\xi^{\prime \prime}\right\|_{L^{2}}\left\|\bar{v}_{n}^{\prime \prime}\right\|_{L^{2}}
$$

Since

$$
\int_{0}^{1}\left(\bar{v}_{n}^{\prime \prime}\right)^{2}=\int_{[0,1]}\left(\psi-w_{n}\right) \mathrm{d} s_{n} \leqq\left\|\left\langle\psi-w_{n}\right\rangle^{+}\right\|_{L^{\infty}} s_{n}\left(\operatorname{supp}\left\langle\psi-w_{n}\right\rangle^{+}\right),
$$

where $\langle x\rangle^{+}=\max \{x, 0\}$, the choice $K=\operatorname{supp}\left\langle\psi-w_{n}\right\rangle^{+}$yields

$$
\int_{0}^{1}\left(\bar{v}_{n}^{\prime \prime}\right)^{2} \leqq\left\|\left\langle\psi-w_{n}\right\rangle^{+}\right\|_{L^{\infty}}\left\|\xi_{1}^{\prime \prime}\right\|_{L^{2}}\left\|\bar{v}_{n}^{\prime \prime}\right\|_{L^{2}}
$$

that is

$$
\left\|\bar{v}_{n}^{\prime \prime}\right\|_{L^{2}} \leqq\left\|\left\langle\psi-w_{n}\right\rangle^{+}\right\|_{L^{\infty}}\left\|\xi_{1}^{\prime \prime}\right\|_{L^{2}}
$$

It then suffices to set $K=[\alpha, \beta]$ to obtain the desired estimate of the total mass of the non-negative measure $s_{n}$ :

$$
s_{n}(] 0,1[)=s_{n}([\alpha, \beta]) \leqq\left\|\xi_{1}^{\prime \prime}\right\|_{L^{2}}\left\|\xi_{2}^{\prime \prime}\right\|_{L^{2}}\left\|\left\langle\psi-w_{n}\right\rangle^{+}\right\|_{L^{\infty}} .
$$

Next, from inequalities (11) and (12), we find that

$$
\left\|\bar{v}_{n}\right\|_{H^{3}} \leqq C,
$$

for some real constant $C$ independent of $n$. Consequently, there exists a subsequence of $\left(\bar{v}_{n}\right)$ converging weakly in $H^{3}$. But in view of (13), this weak limit must be $\bar{v}$. Recalling that the weak topology of a closed ball in a separable Hilbert space is metrizable and that a sequence with values in a compact metric space having a unique cluster value must converge towards it, one can deduce that the whole sequence $\bar{v}_{n}$ converges weakly towards $\bar{v}$ in $H^{3}$. we now propose to prove that this convergence is actually strong. One has

$$
\int_{0}^{1}\left(\bar{v}_{n}^{\prime \prime \prime}-\bar{v}^{\prime \prime \prime}\right)^{2}=-\int_{0}^{1}\left(\bar{v}_{n}^{\prime \prime}-\bar{v}^{\prime \prime}\right)\left(\mathrm{d} s_{n}-\mathrm{d} s\right) .
$$

But, since $\bar{v}_{n}^{\prime \prime}(0)=\bar{v}^{\prime \prime}(0)=0$ and the sequence $\bar{v}_{n}$ converges weakly towards $\bar{v}$ in $H^{3}$, the sequence $\bar{v}_{n}^{\prime \prime}-\bar{v}^{\prime \prime}$ must converge pointwise towards 0 and be bounded by a constant $C$ which is independent of $x$ and $n$. By Egoroff's theorem, there exists a measurable subset $M$ of $[0,1]$ such that the sequence $\bar{v}_{n}^{\prime \prime}-\bar{v}^{\prime \prime}$ converges towards 0 uniformly on $[0,1] \backslash M$, where $s(M)$ is as small as desired. Thus

$$
\int_{[0,1] \backslash M}\left|\bar{v}_{n}^{\prime \prime}-\bar{v}^{\prime \prime}\right|\left(\mathrm{d} s_{n}+\mathrm{d} s\right) \leqq \varepsilon\left[s([0,1])+s_{n}([0,1])\right],
$$


which is controlled by estimate (14). Moreover

$$
\int_{M}\left|\bar{v}_{n}^{\prime \prime}-\bar{v}^{\prime \prime}\right|\left(\mathrm{d} s_{n}+\mathrm{d} s\right) \leqq\left[\left\|\bar{v}_{n}^{\prime \prime}\right\|_{L^{\infty}}+\left\|\bar{v}^{\prime \prime}\right\|_{L^{\infty}}\right]\left[s(M)+s_{n}(M)\right] .
$$

Since $\left\|\bar{v}_{n}^{\prime \prime}\right\|_{L^{\infty}}$ is bounded, the desired conclusion will be reached as soon as

$$
\lim _{n \rightarrow+\infty} s_{n}(M)=s(M),
$$

has been proved. But, it suffices to establish that for all functions $\xi \in C_{0}^{\infty}(] 0,1[)$, one has

$$
\lim _{n \rightarrow+\infty} \int_{] 0,1[} \xi \mathrm{d} s_{n}=\int_{] 0,1[} \xi \mathrm{d} s
$$

And, since

$$
\int_{] 0,1[} \xi \mathrm{d} s_{n}=-\int_{0}^{1} \xi^{\prime} \bar{v}_{n}^{\prime \prime \prime},
$$

this is a consequence of the weak convergence in $H^{3}$ of $\bar{v}_{n}$ towards $\bar{v}$.

\section{Existence of weak solutions and related open problems}

The following example is presented to show that, with the regularity that was proved above of the friction threshold $s$ (Theorems 1 and 3), there may exist no weak solution to the frictional quasi-static problem. Incidentally, this example shows that a sweeping process associated with an arbitrary Wijsman-regulated set-valued mapping need not have any weak solution.

Example. Let us consider the initial condition defined by $u_{0}(x)=1-2|x-1 / 2|$ with $x \in] 0,1[$. The displacements prescribed at the extremities, as well as the body forces, are assumed to vanish identically $u_{0}^{\mathrm{p}} \equiv u_{1}^{\mathrm{p}} \equiv 0, f \equiv 0$. Assuming that the friction coefficient is larger than 2 in order to prevent any slipping, one assumes the measure $s(t)$ to be a "moving Dirac measure" $\delta_{p(t)}$ at position $x=p(t)$. The position $p(t)$ will be an oscillating function around $x=1 / 2$, which is continuous but shows unbounded variation. To define the function $p(t)$, take a sequence $\alpha_{n}$ in ]0,1/4[ converging towards 0 such that $\sum_{n=0}^{\infty} \alpha_{n}=\infty$. Then set

$$
\begin{aligned}
& p(0)=1 / 2, \\
& p(t)=\mid \begin{array}{ll}
1 / 2+(-1)^{n} 2^{2 n+2} \alpha_{n}\left|t-1 / 2^{2 n+2}\right| & \text { if } t \in\left[1 / 2^{2 n+2}, 1 / 2^{2 n+1}\right], \\
1 / 2+(-1)^{n} 2^{2 n+1} \alpha_{n}\left|t-1 / 2^{2 n}\right| & \text { if } t \in\left[1 / 2^{2 n+1}, 1 / 2^{2 n}\right] .
\end{array}
\end{aligned}
$$

It can be readily checked that the support of the measure $\delta_{p(t)}$ is contained in $[1 / 4,3 / 4]$, its total mass equals 1 , and $\delta_{p(t)} \in C^{0}\left([0,1] ; H^{-1}\right)$. From Proposition 1 , it follows that the set-valued mapping $\mathcal{C}(t)$ associated with the underlying sweeping process is Wijsman-regulated. 
Next, set

$$
s_{n}(t)=\mid \begin{array}{ll}
\delta_{1 / 2} & \text { if } t \in\left[0,1 / 2^{2 n}\right. \\
s(t) & \text { if } t \in\left[1 / 2^{2 n}, 1\right],
\end{array}
$$

so that the sweeping process based on the associated $\mathcal{C}_{n}(t)$ admits a weak solution $u_{n}(t)$, which can be explicitly computed. It can easily be checked that for all $m \leqq n$ :

$$
\forall t \geqq \frac{1}{2^{2 m}}, \quad \forall x \in[0,1], \quad 0 \leqq u_{n}(x, t) \leqq \frac{3}{2} \prod_{k=m}^{n}\left(\frac{1-2 \alpha_{k}}{1+2 \alpha_{k}}\right)^{2} .
$$

This estimate entails

$$
\lim _{n \rightarrow \infty} u_{n}(t)=0
$$

at all $t \in] 0,1]$. If we go back to the sweeping process based on $\mathcal{C}(t)$, and taking $u_{P}(t)$ to denote the piecewise constant function associated with a given subdivision $P$ by use of the catching-up algorithm, it can be readily checked that the net $u_{P}(t)$ converges pointwise towards the following function:

$$
u(t)=\mid \begin{array}{ll}
u_{0} & \text { if } t=0, \\
0 & \text { if } t \in] 0,1] .
\end{array}
$$

The convergence cannot be uniform on $[0,1]$, because otherwise the limit would be right-continuous at 0 , in view of Proposition 11. The sweeping process based on $\mathcal{C}(t)$, which was found above to be Wijsman-regulated, therefore does not have any weak solution in the sense of Definition 9.

It might seem that pointwise convergence of the net $u_{P}(t)$ could be allowed by weakening the definition of a weak solution. However, one can model a rigid motion of a segment $\mathcal{C}(t)$ in $\mathbb{R}^{2}$ such that $\mathcal{C}(t)$ is Wijsman-regulated and the corresponding net $u_{P}(t)$ does not converge, even pointwisely. Our Definition 9 of weak solutions of sweeping processes by Wijsman-regulated set-valued mapping therefore seems to be appropriate. However, since a weak solution does not necessarily exist, some problems still remain to be solved.

Open problem 1. Find regularity assumptions about $s(t)$ compatible with a "moving Dirac measure", where the existence of a weak solution to the underlying sweeping process could be proved. Of course, the regularity assumptions will have to be weak enough to be ensured by requiring that the data involved in the "normal problem" show some regularity.

Open problem 2. In cases where regularizing $s(t)$ by performing spatial convolution with a mollifier gives a set-valued mapping $\mathcal{C}(t)$ with bounded retraction, is it true that the corresponding solutions of the associated sweeping processes converge uniformly with $t$ towards a limit? If so and a weak solution of the sweeping process based on $\mathcal{C}(t)$ does exist, are both limits necessarily equal?

Open problem 3. In cases where the sweeping process based on $\mathcal{C}(t)$ admits a weak solution $u(t)$, is it true that $\dot{u}$ is a function of bounded variation of $x$ at every $t$ ? 


\section{Appendix A: Set-valued mappings that are of bounded retraction or Wijsman-regulated}

Let $E$ be an arbitrary metric space whose distance function is denoted by $d$.

Definition 1. The excess of a subset $A$ of $E$ over a subset $B$ is defined as

$$
e(A, B)=\sup _{a \in A} \inf _{b \in B} d(a, b),
$$

where the supremum should be understood with respect to the order on $[0,+\infty]$, so that

$$
\begin{array}{lll}
\forall B \in \mathcal{P}(E), & e(\varnothing, B)=0, \\
\forall A \in \mathcal{P}(E) \backslash\{\varnothing\}, & e(A, \varnothing)=+\infty .
\end{array}
$$

The Hausdorff "distance" between the two subsets $A$ and $B$ of $E$ is defined by

$$
h(A, B)=\max \{e(A, B), e(B, A)\} \in[0,+\infty] .
$$

A key fact, which is recalled in the following proposition, is that the excess gives rise to a triangular inequality.

Proposition 2. For all $A, B, C \subset E$, we have

$$
\begin{aligned}
\text { (i) } & e(A, B)=0 \Longleftrightarrow A \subset \bar{B}, \\
\text { (ii) } & h(A, B)=0 \Longleftrightarrow \bar{A}=\bar{B}, \\
\text { (iii) } & e(A, C) \leqq e(A, B)+e(B, C), \\
\text { (iv) } & h(A, C) \leqq h(A, B)+h(B, C) .
\end{aligned}
$$

The class of all non-empty closed bounded subsets of $E$ equipped with the Hausdorff distance is a metric space. Hence, the Hausdorff distance defines a notion of limit for sequences $C_{n}: \mathbb{N} \rightarrow \mathcal{P}(E)$ of subsets of $E$.

Definition 2. A sequence $C_{n}: \mathbb{N} \rightarrow \mathcal{P}(E)$ of subsets of $E$ will be said to converge in the sense of Hausdorff towards a closed subset $L \subset E$ if

$$
\lim _{n \rightarrow \infty} h\left(C_{n}, L\right)=0 .
$$

In practice, convergence in the sense of Hausdorff is often too strong, as seen in the following example.

Example. In Euclidean $\mathbb{R}^{2}$, let us consider the sequence $C_{n}: \mathbb{N} \rightarrow \mathcal{P}\left(\mathbb{R}^{2}\right)$ defined by

$$
C_{n}=\left\{(x, y) \in \mathbb{R}^{2} \mid y \geqq \frac{x^{2}}{n+1}\right\},
$$

and take $\Pi^{+}$to denote the closed half-space $y \geqq 0$. As:

$$
\forall n \in \mathbb{N}, \quad h\left(C_{n}, \Pi^{+}\right)=+\infty,
$$

the sequence $C_{n}$ does not converge in the sense of Hausdorff towards $\Pi^{+}$. 
Definition 3. Let $C_{n}: \mathbb{N} \rightarrow \mathcal{P}(E)$ be a sequence of subsets of $E$. The two closed sets (possibly empty) defined by

$$
\begin{aligned}
& \liminf _{n \rightarrow \infty} C_{n}=\left\{x \in E \mid \limsup _{n \rightarrow \infty} d\left(x, C_{n}\right)=0\right\}, \\
& \limsup _{n \rightarrow \infty} C_{n}=\left\{x \in E \mid \liminf _{n \rightarrow \infty} d\left(x, C_{n}\right)=0\right\},
\end{aligned}
$$

always satisfy:

$$
\liminf _{n \rightarrow \infty} C_{n} \subset \limsup _{n \rightarrow \infty} C_{n} .
$$

When these two sets equal a set $L$ (necessarily closed), it will be said that the sequence $C_{n}$ converges in the sense of Kuratowski towards $L$, which will be written:

$$
\lim _{n \rightarrow \infty} C_{n}=L .
$$

Definition 4. A sequence $C_{n}: \mathbb{N} \rightarrow \mathcal{P}(E)$ of subsets of $E$ will be said to converge in the sense of Wijsman towards a closed set $L \subset E$ if

$$
\forall x \in E, \quad \lim _{n \rightarrow \infty} d\left(x, C_{n}\right)=d(x, L) .
$$

The interest of convergence in the sense of Wijsman is that it is induced by a natural topology in the class of all nonempty closed subsets of $E$ : the weak topology generated by the family of functions $d(x, \cdot)$, when $x$ covers $E$, which is called Wijsman's topology.

Theorem 4. [4] Let $(E, d)$ be a complete separable metric space. Then the class of nonempty closed subsets of E equipped with Wijsman's topology is separable, and there is a complete metric compatible with the topology.

A link between convergence in the sense of Hausdorff and convergence in the sense of Kuratowski is provided by the following proposition (a proof of which can be found in [9]).

Proposition 3. Let $C_{n}: \mathbb{N} \rightarrow \mathcal{P}(E)$ be a sequence of subsets of $E$, and $L$ a closed set. If $C_{n}$ converges towards $L$ in the sense of Hausdorff, then $C_{n}$ converges towards $L$ in the sense of Kuratowski:

$$
\lim _{n \rightarrow \infty} h\left(C_{n}, L\right)=0 \Longrightarrow \lim _{n \rightarrow \infty} C_{n}=L .
$$

If all the $C_{n}$ are contained in a fixed compact set $K \subset E\left(\forall n \in \mathbb{N}, C_{n} \subset K\right)$, then the converse is true.

A link between convergence in the sense of Kuratowski and convergence in the sense of Wijsman is provided by the following proposition.

Proposition 4. Let $C_{n}: \mathbb{N} \rightarrow \mathcal{P}(E)$ be a sequence of subsets of $E$, and $L$ a closed set. If $C_{n}$ converges towards $L$ in the sense of Wijsman, then $C_{n}$ will converge towards $L$ in the sense of Kuratowski. 
Proof. This is a straightforward consequence of following two simple statements:

$$
\begin{aligned}
& \forall x \in E, \quad d(x, L) \geqq \limsup _{n \rightarrow \infty} d\left(x, C_{n}\right) \quad \Longrightarrow \quad L \subset \liminf _{n \rightarrow \infty} C_{n}, \\
& \forall x \in E, \quad d(x, L) \leqq \liminf _{n \rightarrow \infty} d\left(x, C_{n}\right) \quad \Longrightarrow \quad L \supset \limsup _{n \rightarrow \infty} C_{n} .
\end{aligned}
$$

Definition 5. [9] A set-valued mapping $C:\left[t_{0}, T\right] \rightarrow \mathcal{P}(E)$ will be said to have bounded retraction if

$$
\operatorname{ret}\left(C ; t_{0}, T\right) \stackrel{\text { def }}{=} \sup \sum_{i=1}^{n} e\left(C\left(t_{i-1}\right), C\left(t_{i}\right)\right)<\infty \text {, }
$$

where the supremum is taken over all the finite sequences $t_{0} \leqq t_{1} \leqq t_{2} \leqq \cdots \leqq$ $t_{n}=T$. The function $t \mapsto \operatorname{ret}\left(C ; t_{0}, t\right)$ thus defined is non-decreasing.

Theorem 5. [9] Let $C:\left[t_{0}, T\right] \rightarrow \mathcal{P}(E)$ be a set-valued mapping with bounded retraction. Then, $C(t)$ admits a left limit $C(t-)$ in the sense of Kuratowski at every $\left.t \in] t_{0}, T\right]$, and a right limit $C(t+)$, at every $t \in\left[t_{0}, T[\right.$.

Definition 6. A set-valued mapping $C:\left[t_{0}, T\right] \rightarrow \mathcal{P}(E)$ will be said to have absolutely continuous retraction if, for all $\varepsilon>0$, some $\eta>0$ can be found such that for all finite collection $] \sigma_{i}, \tau_{i}\left[\subset\left[t_{0}, T\right]\right.$ of non-overlapping open intervals, the following statement:

$$
\sum_{i}\left(\tau_{i}-\sigma_{i}\right)<\eta \Longrightarrow \sum_{i} e\left(C\left(\sigma_{i}\right), C\left(\tau_{i}\right)\right)<\varepsilon
$$

holds true, and to show Lipschitz-continuous retraction if there exists $L \geqq 0$ such that

$$
\forall s \leqq t \in\left[t_{0}, T\right], \quad e(C(s), C(t)) \leqq L(t-s)
$$

The following proposition accounts for the terminology used here.

Proposition 5. [9] Let $C:\left[t_{0}, T\right] \rightarrow \mathcal{P}(E)$ be a set-valued mapping. The following two claims are then equivalent:

(i) C has absolutely continuous (respectively Lipschitz-continuous) retraction.

(ii) $C$ has bounded retraction and the non-decreasing real-valued function $\tau \mapsto$ $\operatorname{ret}\left(C ; t_{0}, \tau\right)$ is absolutely continuous (respectively Lipschitz-continuous).

On similar lines, we have the following proposition:

Proposition 6. [9] Let $C:\left[t_{0}, T\right] \rightarrow \mathcal{P}(E)$ be a set-valued mapping with bounded retraction. The following three claims are then equivalent:

(i) $C$ has right-continuous retraction at $t \in\left[t_{0}, T[\right.$ (that is, the real-valued function $\tau \mapsto \operatorname{ret}\left(C ; t_{0}, \tau\right)$ is right-continuous at $\left.t\right)$.

(ii) $\lim _{\tau \rightarrow t+} e(C(t), C(\tau))=0$.

(iii) $C(t) \subset C(t+)$. 
Classically, a function $f:\left[t_{0}, T\right] \rightarrow E$ is said to be regulated if there is a sequence of step functions converging towards $f$ uniformly with regard to $t \in$ $\left[t_{0}, T\right]$. In the specific case where the metric space is complete, a function $f$ : $\left[t_{0}, T\right] \rightarrow E$ is regulated if and only if it admits a left limit $f(t-)$ at every $\left.\left.t \in\right] t_{0}, T\right]$ and a right limit $f(t+)$ at every $t \in\left[t_{0}, T[\right.$.

Definition 7. A set-valued mapping $C:\left[t_{0}, T\right] \rightarrow \mathcal{P}(E)$ with non-empty closed values, will be said to be Wijsman-regulated if it is regulated as a mapping with values in the class of nonempty closed subsets of $E$ equipped with Wijsman's topology.

In what follows, only the specific case where the metric space $(E, d)$ is a separable Hilbert space $H$ will be considered. The scalar product will be denoted by $(\cdot \mid \cdot)$, the norm by $\|\cdot\|$ and the closed ball with center $c$ and radius $r$ by $B(c, r)$. The notation $\mathcal{C}(H)$ will stand for the class consisting of the non-empty closed convex subsets of $H$.

Theorem 6. Let $C_{n}: \mathbb{N} \rightarrow \mathcal{C}(H)$ be a sequence of nonempty closed convex subsets of $H$. If this sequence has a non-empty limit $L$ in the sense of Kuratowski, then $L$ is convex, and the following statement holds true:

$$
\forall x \in H, \quad \lim _{n \rightarrow \infty} \operatorname{proj}\left[x, C_{n}\right]=\operatorname{proj}[x, L] .
$$

Proof. Fix $x \in H$ arbitrary and set

$$
\begin{aligned}
x_{n} & =\operatorname{proj}\left[x, C_{n}\right], \\
l & =\operatorname{proj}[x, L] .
\end{aligned}
$$

It has to be proved that the sequence $\left(x_{n}\right)$ converges strongly towards $l$. Let $c \in L$ be arbitrary. The definition of $\lim _{n \rightarrow \infty} C_{n}$ (convergence in the sense of Kuratowski) gives:

$$
\forall m \in \mathbb{N}, \quad \exists N_{c, m} \in \mathbb{N}, \quad \forall n \geqq N_{c, m}, \quad d\left(c, C_{n}\right)<\frac{1}{m+1} .
$$

Setting $c=l, m=0$ and removing finitely many terms of the sequence, if necessary, we obtain

$$
d\left(l, C_{n}\right)<1 .
$$

Hence, the sequence $\left(x_{n}\right)$ takes values in the closed ball having center $x$ and radius $1+2\|l-x\|$. Therefore, a subsequence, still denoted by $\left(x_{n}\right)$, converges weakly towards $\tilde{l} \in B(x, 1+2\|l-x\|)$.

Next, fix $c \in L$ and $m \in \mathbb{N}$ arbitrarily. From statement (15), we can find $N \in \mathbb{N}$ such that

$$
\forall n \geqq N, \quad \exists b_{n} \in B(0,1), \quad c+\frac{b_{n}}{m+1} \in C_{n} .
$$


With $n \geqq N$, we obtain

$$
\left(x-x_{n} \mid c+\frac{b_{n}}{m+1}-x_{n}\right) \leqq 0,
$$

; therefore,

$$
\left(x-x_{n} \mid c-x_{n}\right) \leqq \frac{1+2\|l-x\|}{m+1} .
$$

Taking the infimum limit $n \rightarrow \infty$ in this inequality, one obtains

$$
(x-\tilde{l} \mid c)-(x \mid \tilde{l})+\liminf _{n \rightarrow \infty}\left\|x_{n}\right\|^{2} \leqq \frac{1+2\|l-x\|}{m+1} ;
$$

therefore,

$$
\forall m \in \mathbb{N}, \quad \forall c \in L, \quad(x-\tilde{l} \mid c-\tilde{l})_{H} \leqq \frac{1+\|l-x\|}{m+1},
$$

which yields

$$
\tilde{l}=l
$$

because of the uniqueness of the projection of a point onto a closed convex subset of a Hilbert space. Remembering that the weak topology in a closed ball of a separable Hilbert space is metrizable and that a sequence in a compact metric space that has a unique cluster value converges towards it, it has been actually proved that the whole sequence converges weakly towards $l$ (with no need to extract any subsequences).

Finally, since $\left\|x-x_{n}\right\|=d\left(x, C_{n}\right)$, setting $c=l$ in statement (15) yields

$$
\forall m \in \mathbb{N}, \quad \exists N_{m} \in \mathbb{N}, \quad \forall n \geqq N_{m}, \quad\left\|x-x_{n}\right\| \leqq\|x-l\|+\frac{1}{m+1},
$$

and therefore

$$
\limsup _{n \rightarrow \infty}\left\|x-x_{n}\right\| \leqq\|x-l\|,
$$

which suffices to ensure that the weak convergence of the sequence $\left(x_{n}\right)$ is actually a strong convergence.

Corollary 1. Let $C_{n}: \mathbb{N} \rightarrow \mathcal{C}(H)$ be a sequence of non-empty closed convex subsets of $H$, and $L \in \mathcal{C}(H)$. The following three statements are then equivalent.

$$
\begin{array}{ll}
\text { (i) } & \lim _{n \rightarrow \infty} C_{n}=L, \\
\text { (ii) } & \forall x \in H, \quad \lim _{n \rightarrow \infty} d\left(x, C_{n}\right)=d(x, L), \\
\text { (iii) } & \forall x \in H, \quad \lim _{n \rightarrow \infty} \operatorname{proj}\left[x, C_{n}\right]=\operatorname{proj}[x, L] .
\end{array}
$$


Proof. The identity:

$$
d\left(x, C_{n}\right)=d\left(x, \operatorname{proj}\left[x, C_{n}\right]\right),
$$

gives (iii) $\Rightarrow$ (ii), Proposition 4 , (ii) $\Rightarrow$ (i), and finally, Theorem 6 is exactly (i) $\Rightarrow$ (iii).

In particular, with sequences of non-empty closed, convex subsets in a separable Hilbert space, convergence in the sense of Kuratowski and in the sense of Wijsman is the same. In the specific case of finite-dimensional Hilbert spaces, this fact was first proved by Wijsman in 1966 (see [12]) for sequences of non-empty closed subsets which are not necessarily convex. Corollary 1 is simply a particular case of more general extensions of Wijsman's theorem to infinite dimensions which were reviewed in [5] in 1994. The aim of the following example is to show that in an infinite-dimensional Hilbert space, the additional assumption of convexity cannot be relaxed.

Example. Take $e_{n}$ to denote the vectors of the canonical basis of $l^{2}$. For all $n \in \mathbb{N}$, set

$$
C_{n}=\left\{2 e_{0}, e_{n}\right\}, \quad L=\left\{2 e_{0}\right\} .
$$

It can be readily checked that

$$
\lim _{n \rightarrow \infty} C_{n}=L,
$$

but

$$
d\left(0, C_{n}\right)=1, \quad d(0, L)=2 .
$$

Proposition 7. Let $C:\left[t_{0}, T\right] \rightarrow \mathcal{C}(H)$ be an arbitrary set-valued mapping with non-empty closed convex values. The following three statements are then equivalent:

(i) $C$ is Wijsman-regulated.

(ii) $C$ admits a non-empty left limit in the sense of Kuratowski (notation $C(t-)$ ) at every $\left.t \in] t_{0}, T\right]$ and a non-empty right limit (notation $C(t+)$ ) at every $t \in\left[t_{0}, T[\right.$.

(iii) For all $x \in H$, the mapping

$$
\left\{\begin{aligned}
{\left[t_{0}, T\right] } & \rightarrow H \\
t & \mapsto \operatorname{proj}[x, C(t)]
\end{aligned}\right.
$$

is regulated.

Proof. This is straightforward consequence of Theorem 4 and Corollary 1.

We are now able to list some classes of Wijsman-regulated set-valued mappings.

Proposition 8. Let $C:\left[t_{0}, T\right] \rightarrow \mathcal{C}(H)$ be a set-valued mapping with bounded retraction, the values $C(t)$ of which are non-empty closed convex, at all $t \in\left[t_{0}, T\right]$. Then $C$ is Wijsman-regulated. 
Proof. This is a straightforward consequence of Theorem 5 and Proposition 7.

Hence, in the case of set-valued mappings with non-empty closed convex values in a Hilbert space, the class consisting of the Wijsman-regulated set-valued mappings contains the class consisting of the set-valued mappings with bounded retraction. Another important class of Wijsman-regulated set-valued mappings is that of those set-valued mappings that are regulated in the sense of Hausdorff distance.

Theorem 7. A set-valued mapping $C:\left[t_{0}, T\right] \rightarrow \mathcal{C}(H)$ is said to be regulated in the sense of the Hausdorff distance if there exists a sequence $C_{n}:\left[t_{0}, T\right] \rightarrow \mathcal{P}(H)$ of piecewise constant set-valued mappings such that the sequence of real-valued functions $t \mapsto h\left(C_{n}(t), C(t)\right)$ converges uniformly towards 0 .

Any set-valued mapping $C:\left[t_{0}, T\right] \rightarrow \mathcal{C}(H)$ which is regulated in the sense of the Hausdorff distance is Wijsman-regulated. Moreover, in those cases where the values of $C$ are contained in a fixed compact subset $K \subset H\left(\forall t \in\left[t_{0}, T\right], C(t) \subset\right.$ $K)$, then the converse is true.

\section{Proof. Necessary condition.}

Let us consider a set-valued mapping $C:\left[t_{0}, T\right] \rightarrow \mathcal{C}(H)$ which is regulated in the sense of the Hausdorff distance. Based on Proposition 7, the conclusion targeted will be reached if at an arbitrary $t \in\left[t_{0}, T\left[\right.\right.$, it can be proved that $\lim _{i n f} \inf _{\tau \rightarrow+} C(\tau) \neq \varnothing$ and $\liminf \operatorname{int+}_{\tau \rightarrow} C(\tau)=\limsup _{\tau \rightarrow t+} C(\tau)$.

- First let us prove that the infimum limit is non-empty. There exists a piecewise constant set-valued mapping $C_{n_{0}}$ such that

$$
\forall t \in\left[t_{0}, T\right], \quad h\left(C_{n_{0}}(t), C(t)\right) \leqq \frac{1}{2},
$$

and a finite collection $\left\{a_{k}\right\}$ of elements of $H$ such that all the $C_{n_{0}}(t)$ contain at least one of the $a_{k}$. Let $B$ be a closed ball with center $a_{0}$ and a radius larger than 2 plus the maximum of the distance from $a_{0}$ to one of the $a_{k}$. Then, for all $n \in \mathbb{N}$, there exists a piecewise constant set-valued mapping $C_{n}:\left[t_{0}, T\right] \rightarrow \mathcal{P}(H)$ such that

$$
\forall t \in\left[t_{0}, T\right], \quad h\left(C_{n}(t), C(t)\right) \leqq \frac{1}{n+1}, \quad \text { and } \quad B \cap C_{n}(t) \neq \varnothing .
$$

This entails

$$
\begin{aligned}
& \forall n \in \mathbb{N}, \quad \exists x_{n} \in B, \quad \exists \eta_{n}>0, \\
& \forall \tau \in] t, t+\eta_{n}\left[, \quad d\left(x_{n}, C(\tau)\right)<\frac{1}{n+1} .\right.
\end{aligned}
$$

One can then extract a subsequence, which is still written $\left(x_{n}\right)$, that converges weakly towards $l \in B$. It is now proposed to prove that

$$
l \in \liminf _{\tau \rightarrow t+} C(\tau),
$$


that is

$$
\lim _{\tau \rightarrow t+} d(l, C(\tau))=0 .
$$

Fix $m \in \mathbb{N}$. Based on Mazur's theorem, there exists a convex combination $c_{m}$ of the $x_{n}$ such that $d\left(l, c_{m}\right)<1 /(m+1)$. Since all the $x_{n}$ in that convex combination can be chosen with arbitrarily large ranks, one can assume

$$
\exists \eta>0, \quad \forall \tau \in] t, t+\eta\left[, \quad d\left(x_{n}, C(\tau)\right)<\frac{1}{m+1},\right.
$$

for all the $x_{n}$ in that convex combination. In addition, the convexity of $C(\tau)+$ $B(0,1 /(m+1))$ entails

$$
\forall \tau \in] t, t+\eta\left[, \quad d\left(c_{m}, C(\tau)\right)<\frac{1}{m+1},\right.
$$

and the conclusion targeted is reached, since $d(l, C(\tau)) \leqq d\left(l, c_{m}\right)+$ $d\left(c_{m}, C(\tau)\right)$.

- It still remains to be proved that the infimum limit equals the supremum limit. Let $h \in \lim \sup _{\tau \rightarrow t+} C(\tau)$, and $\varepsilon>0$. Since $C(t)$ is regulated in the sense of Hausdorff distance, one can find a set $C_{m} \subset H$ and a real number $\eta>0$ such that

$$
\forall \tau \in] t, t+\eta\left[, \quad h\left(C_{m}, C(\tau)\right)<\frac{\varepsilon}{3} .\right.
$$

Since $h \in \lim \sup _{\tau \rightarrow t+} C(\tau)$,

$$
\left.\exists \tau^{\prime} \in\right] t, t+\eta\left[, \quad d\left(h, C\left(\tau^{\prime}\right)\right)<\frac{\varepsilon}{3} .\right.
$$

Therefore, for all $\tau \in] t, t+\eta[$,

$$
d(h, C(\tau)) \leqq d\left(h, C\left(\tau^{\prime}\right)\right)+h\left(C\left(\tau^{\prime}\right), C_{m}\right)+h\left(C_{m}, C(\tau)\right)<\varepsilon,
$$

which proves that $h \in \liminf _{\tau \rightarrow t+} C(\tau)$.

\section{Sufficient condition.}

Let $C(t)$ be a Wijsman-regulated set-valued mapping with values contained in a fixed compact set. By using both Propositions 7 and 3, this set-valued mapping admits left and right limits in the sense of Hausdorff at every $t$. Therefore, choosing $n \in \mathbb{N}$ and $t \in\left[t_{0}, T\right]$ arbitrarily, one obtains

$$
\begin{array}{ll}
\exists \eta_{t}>0, & \forall \tau \in] t-\eta_{t}, t[, \quad h(C(\tau), C(t-))<1 /(n+1), \\
\forall \tau \in] t, t+\eta_{t}[, \quad h(C(\tau), C(t+))<1 /(n+1) .
\end{array}
$$

From the open sets $] t-\eta_{t}, t+\eta_{t}$ [ defining a covering of the compact $\left[t_{0}, T\right]$, a finite subcovering defined by $t_{0}<t_{1}<t_{2}<\cdots<t_{n}=T$ can be extracted. Let us define a piecewise constant set-valued mapping $C_{n}$ by

$$
\left.\forall i, \quad C_{n}\left(t_{i}\right)=C\left(t_{i}\right), \quad \text { et } \forall i, \forall \tau \in\right] t_{i-1}, t_{i}\left[, \quad C_{n}(\tau)=C\left(\frac{t_{i-1}+t_{i}}{2}\right) .\right.
$$


From this definition, for all $\tau \in] t_{i-1}, t_{i}[$, one obtains

$$
\begin{aligned}
h & \left(C_{n}(\tau), C(\tau)\right) \\
& \leqq h\left(C(\tau), C\left(t_{i}-\right)\right)+h\left(C\left(t_{i}-\right), C_{n}\left(t_{i}-\right)\right)+h\left(C_{n}\left(t_{i}-\right), C_{n}(\tau)\right), \\
& \quad<\frac{1}{n+1}+\frac{1}{n+1}+0=\frac{2}{n+1},
\end{aligned}
$$

which shows that $C(t)$ is regulated in the sense of the Hausdorff distance.

Corollary 2. Every set-valued mapping $C:\left[t_{0}, T\right] \rightarrow \mathcal{C}(H)$ which is continuous in the sense of the Hausdorff distance:

$$
\forall \varepsilon>0, \quad \exists \eta>0, \quad \forall \tau \in] t-\eta, t+\eta[, \quad h(C(\tau), C(t))<\varepsilon,
$$

is Wijsman-regulated.

Another class of Wijsman-regulated set-valued mappings is provided by the class of non-increasing set-valued mappings with non-empty closed convex values.

Proposition 9. Let $C:\left[t_{0}, T\right] \rightarrow \mathcal{C}(H)$ be a set-valued mapping with non-empty closed convex values, which is assumed to be non-increasing in the sense that

$$
\forall t_{1}, t_{2} \in\left[t_{0}, T\right], \quad t_{1} \leqq t_{2} \Longrightarrow C\left(t_{2}\right) \subset C\left(t_{1}\right)
$$

Then $C(t)$ is Wijsman-regulated.

Proof. It can be readily checked that

$$
\begin{aligned}
& \limsup _{\tau \rightarrow t-} C(\tau) \subset \bigcap_{\tau \in\left[t_{0}, t[\right.} C(\tau) \subset \liminf _{\tau \rightarrow t-} C(\tau), \\
& \limsup _{\tau \rightarrow t+} C(\tau) \subset \bigcup_{\tau \in] t, T]} C(\tau) \subset \liminf _{\tau \rightarrow t+} C(\tau),
\end{aligned}
$$

which shows that $C$ admits the left and right limits:

$$
\begin{aligned}
& C(t-)=\bigcap_{\tau \in\left[t_{0}, t[\right.} C(\tau), \\
& C(t+)=\frac{\bigcup_{\tau \in] t, T]} C(\tau),}{}
\end{aligned}
$$

which are non-empty since they contain $C(T)$. Proposition 7 now yields the conclusion targeted. 


\section{Appendix B: Weak solutions of sweeping processes}

In this appendix, $H$ is a separable Hilbert space, and all the set-valued mappings $C:\left[t_{0}, T\right] \rightarrow \mathcal{C}(H)$ will be assumed to take only non-empty closed convex values.

Given a closed convex subset $K$ of $H, \partial I_{K}$ will denote the subdifferential of the indicatrix function (in the sense of convex analysis) of $K$. Hence, $\partial I_{K}(x)$ is the cone of all the outward normals to $K$ at $x$. It will be empty if $x \notin K$ and reduces to $\{0\}$ at any interior point $x$ of $K$. Given a set-valued mapping $C:\left[t_{0}, T\right] \rightarrow \mathcal{C}(H)$ with non-empty closed convex values, we will use the term "sweeping process" to refer to the evolution problem consisting of finding a function $u:\left[t_{0}, T\right] \rightarrow H$ such that

$$
\begin{aligned}
& \text { - } u\left(t_{0}\right)=u_{0}, \\
& \text { - }-\dot{u}(t) \in \partial I_{C(t)}[u(t)], \quad \forall t \in\left[t_{0}, T\right],
\end{aligned}
$$

where $u_{0}$ denotes a given initial condition. This evolution problem has a clear geometrical interpretation in kinematic terms when $C(t)$ has a non-empty interior. As long as the point $u(t)$ is an interior point in the moving convex set $C(t)$, it will remain at rest. When, by the evolution of $C(t)$, the point $u(t)$ meets the boundary of $C(t)$ at some instant $t$, it proceeds in an inward normal direction, so as to go on belonging to $C(t)$, exactly as if it were being pushed by the boundary of the moving convex set.

A definition of weak solutions of sweeping processes was first proposed by MoREAU [10] in the case of set-valued mappings with bounded retraction. He proved their existence before showing that they are actually strong solutions in some sense. In the problems analysed in the present paper, some sweeping processes appear that have weak solutions that are not strong solutions. Of course, the underlying set-valued mappings do not have bounded retraction. Thus, one is led to extend Moreau's definition of weak solutions of sweeping processes to a larger class of set-valued mappings than that showing bounded retraction. Since these set-valued mappings must have a right limit $C(t+)$ in the sense of Kuratowski, at every $t$, one is naturally led to consider the larger class consisting of all the Wijsman-regulated set-valued mappings.

In this appendix, we first define weak solutions of sweeping processes based on Wijsman-regulated set-valued mappings, and these weak solutions, when they exist, are proved to enjoy the same general properties as those of the weak solutions of sweeping processes based on set-valued mappings with bounded retraction. MOREAU's [10] existence results obtained in the case of set-valued mappings with bounded retraction are then briefly recalled without going into the proofs.

Definition 8. We define $P$ as a subdivision of the real interval $\left[t_{0}, T\right]$ (notation $\left.P \in \operatorname{subd}\left(\left[t_{0}, T\right]\right)\right)$ if it is a finite partition of $\left[t_{0}, T\right]$ into intervals of any sort (some of them possibly reduced to single points).

A $P^{\prime} \in \operatorname{subd}\left(\left[t_{0}, T\right]\right)$ will be said to be a refinement of $P \in \operatorname{subd}\left(\left[t_{0}, T\right]\right)$ (notation $P^{\prime} \succ P$ ) if every interval of $P^{\prime}$ is contained in an interval of $P$.

A mapping defined on $\left[t_{0}, T\right]$ will be said to be piecewise constant if it is constant in every interval of some $P \in \operatorname{subd}\left(\left[t_{0}, T\right]\right)$. 
Definition 9. Let $C:\left[t_{0}, T\right] \rightarrow \mathcal{C}(H)$ be a Wijsman-regulated set-valued mapping taking non-empty closed convex values. For $P \in \operatorname{subd}\left(\left[t_{0}, T\right]\right), I_{0}, I_{1}, I_{2}, \ldots$ will denote the ordered sequence of the corresponding intervals, and $t_{i}$ the origin (left extremity) of $I_{i}$. We will also take $C_{P}$ to denote the piecewise constant set-valued mapping with non-empty closed convex values defined by

$$
C_{P}\left(I_{i}\right)=C_{i}=\mid \begin{array}{ll}
C\left(t_{i}\right) & \text { if } t_{i} \in I_{i}, \\
C\left(t_{i}+\right) & \text { if } t_{i} \notin I_{i} .
\end{array}
$$

Given the initial value $a \in C\left(t_{0}\right)$, set inductively ("catching-up" algorithm):

$$
\begin{aligned}
u_{0} & =a, \\
u_{i+1} & =\operatorname{proj}\left(u_{i}, C_{i+1}\right),
\end{aligned}
$$

to define the piecewise constant function $u_{P}:\left[t_{0}, T\right] \rightarrow H$ by

$$
u_{P}\left(I_{i}\right)=u_{i}
$$

When the net $\left(u_{P}\right)$ converges uniformly in $\left[t_{0}, T\right]$, towards some limit $u:\left[t_{0}, T\right] \rightarrow$ $H$ in the sense that

$$
\begin{aligned}
& \forall \varepsilon>0, \quad \exists P \in \operatorname{subd}\left(\left[t_{0}, T\right]\right), \quad \forall P^{\prime} \succ P, \\
& \forall t \in\left[t_{0}, T\right], \quad\left\|u_{P^{\prime}}(t)-u(t)\right\| \leqq \varepsilon,
\end{aligned}
$$

the function $u:\left[t_{0}, T\right] \rightarrow H$ will be said to be a weak solution of the sweeping process based on the set-valued mapping $C(t)$, starting at initial condition $a$.

Proposition 10. Let $C:\left[t_{0}, T\right] \rightarrow \mathcal{C}(H)$ be a Wijsman-regulated set-valued mapping, and $u, u^{\prime}$ be two weak solutions of the associated sweeping process. Then, the real-valued function

$$
\left\{\begin{aligned}
{\left[t_{0}, T\right] } & \rightarrow \mathbb{R}^{+} \\
t & \mapsto\left\|u(t)-u^{\prime}(t)\right\|
\end{aligned}\right.
$$

is non-increasing.

Proof. If $u$ and $u^{\prime}$ start at initial values $a$ and $a^{\prime}$, these functions are the limits of (generalized) sequences $u_{P}$ and $u_{P}^{\prime}$ of the piecewise constant functions inductively defined from these initial data. As the successive values of $u_{P}$ and $u_{P}^{\prime}$ are obtained by performing projections onto the convex sets $C_{i}$, the contraction property of such projections entails that

$$
\forall P \in \operatorname{subd}\left(\left[t_{0}, T\right]\right), \quad \forall s \leqq t, \quad\left\|u_{P}(t)-u_{P}^{\prime}(t)\right\| \leqq\left\|u_{P}(s)-u_{P}^{\prime}(s)\right\| .
$$

It then suffices to go to the limit of the two members of this inequality to obtain the conclusion required. 
Proposition 11. Let $u:\left[t_{0}, T\right] \rightarrow H$ be a weak solution of the sweeping process based on the set-valued mapping $C(t)$, which is assumed to be Wijsman-regulated. Then $u$ admits a left limit $u(t-)$ and a right limit $u(t+)$ at every $t \in\left[t_{0}, T\right]$ (with appropriate adjustments at $t_{0}$ and $T$ ) and

$$
\begin{aligned}
& \forall t \in\left[t_{0}, T\right], \quad u(t) \in C(t), \\
& \left.\forall t \in] t_{0}, T\right], \quad u(t)=\operatorname{proj}(u(t-), C(t)), \\
& \forall t \in\left[t_{0}, T[, \quad u(t+)=\operatorname{proj}(u(t), C(t+)) .\right.
\end{aligned}
$$

Proof. The existence of $u(t-)$ and $u(t+)$ is ensured by the fact that $u$ is regulated.

At an arbitrary $t \in\left[t_{0}, T\right]$, we take $\mathcal{P}$ to denote the set of all subdivisions in $\operatorname{subd}\left(\left[t_{0}, T\right]\right)$ containing $\{t\}$. Based on the definition of $C_{P}$,

$$
\forall P \in \mathcal{P}, \quad C_{P}(t)=C(t),
$$

and therefore, based on the definition of $u_{P}$ :

$$
\forall P \in \mathcal{P}, \quad u_{P}(t)=\operatorname{proj}\left[u_{P}(t-), C(t)\right],
$$

which entails

$$
\forall P \in \mathcal{P}, \quad u_{P}(t) \in C(t)
$$

Taking a limit with respect to $P \in \mathcal{P}$, one can readily see that $u(t) \in C(t)$.

As the convergence of the net $u_{P}, P \in \mathcal{P}$, is uniform with $t$, the following commutation of limits holds:

$$
u(t-)=\lim _{P \in \mathcal{P}} u_{P}(t-),
$$

and therefore,

$$
u(t)=\operatorname{proj}(u(t-), C(t))
$$

Likewise

$$
\forall P \in \mathcal{P}, \quad u_{P}(t+)=\operatorname{proj}\left(u_{P}(t), C(t+)\right),
$$

and the last statement in the proposition can be proved in the same way.

The two following propositions display the local character of the concept of weak solutions.

Proposition 12. Let $u:\left[t_{0}, T\right] \rightarrow H$ be a weak solution of the sweeping process based on $C(t)$. Let $\left[t_{0}^{\prime}, T^{\prime}\right]$ be a subinterval of $\left[t_{0}, T\right]$. Then $u_{\mid\left[t_{0}^{\prime}, T^{\prime}\right] \text {. will be a weak }}$ solution of the sweeping process based on $C_{\mid\left[t_{0}^{\prime}, T^{\prime}\right]}$.

Proposition 13. Let $I_{0}, I_{1}, I_{2}, \ldots$ be a subdivision of $\left[t_{0}, T\right]$ into intervals containing their respective origins $t_{0}, t_{1}, t_{2}, \ldots$, and $u:\left[t_{0}, T\right] \rightarrow H$ a function such that 
(i) For all $i, u_{\mid I_{i}}$ is a weak solution of the sweeping process based on $C_{\mid \bar{I}_{i}}$ (which entails the existence of $u\left(t_{i}-\right)$ for $\left.i>0\right)$.

(ii) For $i>0$ :

$$
u\left(t_{i}\right)=\operatorname{proj}\left(u\left(t_{i}-\right), C\left(t_{i}\right)\right) .
$$

Then $u$ is a weak solution of the sweeping process based on $C$ in $\left[t_{0}, T\right]$.

The following theorem is due to Moreau. It claims that provided the set-valued mapping has bounded retraction, the corresponding sweeping process admits a weak solution starting from any arbitrary initial condition.

Theorem 8. [10] Let $C:\left[t_{0}, T\right] \rightarrow \mathcal{C}(H)$ be a set-valued mapping with nonempty closed convex values, which is assumed to have bounded retraction. Then there exists a weak solution $u$ of the sweeping process starting at any given initial condition $a \in C\left(t_{0}\right)$. This weak solution is such that

$$
\forall s \leqq t \in\left[t_{0}, T\right], \quad\|u(t)-u(s)\| \leqq \operatorname{ret}(C ; s, t) .
$$

In particular, the function $u$ has bounded variation. If, in addition, $C(t)$ has rightcontinuous (respectively absolutely continuous, respectively Lipschitz-continuous) retraction, then the weak solution $u$ is right-continuous (respectively, absolutely continuous, respectively Lipschitz-continuous).

This weak solution depends continuously on the data (the set-valued mapping $C(t)$ and the initial condition) involved in the sweeping process in the sense displayed by the following theorem.

Theorem 9. [10] Let $C, C^{\prime}:\left[t_{0}, T\right] \rightarrow \mathcal{C}(H)$ be two set-valued mappings with non-empty closed convex values and bounded retraction. Then every pair $\left(u, u^{\prime}\right)$ of weak solutions of the associated sweeping processes will satisfy the following estimate:

$$
\begin{aligned}
\forall t & \in\left[t_{0}, T\right], \quad\left\|u(t)-u^{\prime}(t)\right\|^{2}-\left\|u\left(t_{0}\right)-u^{\prime}\left(t_{0}\right)\right\|^{2} \\
& \leqq\left[\sup _{\tau \in\left[t_{0}, t\right]} h\left(C(\tau), C\left(\tau^{\prime}\right)\right)\right]\left[\operatorname{ret}\left(C ; t_{0}, t\right)+\operatorname{ret}\left(C^{\prime} ; t_{0}, t\right)\right] .
\end{aligned}
$$

Theorem 9 can be used to obtain an estimate of the error occurring when the catching-up algorithm is used to approximate the weak solution of a sweeping process with bounded retraction.

Proposition 14. [10] Let $C:\left[t_{0}, T\right] \rightarrow \mathcal{C}(H)$ be a set-valued mapping with nonempty closed convex values and bounded retraction. Consider an arbitrary subdivision $P \in \operatorname{subd}\left(\left[t_{0}, T\right]\right)$ of the interval $\left[t_{0}, T\right]$, let $I_{0}, I_{1}, I_{2}, \ldots$ be the corresponding finite sequence of intervals, and $\mu$ be some majorant of $\operatorname{ret}(C ; s, t)$, for arbitrary $[s, t] \in I_{i}$. Still denoting by $u_{P}$ the piecewise constant function provided by the catching-up algorithm, one has

$$
\left\|u(t)-u_{P}(t)\right\| \leqq 2 \sqrt{\mu \operatorname{ret}\left(C ; t_{0}, t\right)} .
$$


Any function $u \in B V\left(\left[t_{0}, T\right], H\right)$ is classically associated with its differential measure or Stieltjes measure $\mathrm{d} u \in \mathcal{M}\left(\left[t_{0}, T\right], H\right)$. It satisfies, in particular,

$$
\int_{] s, t]} \mathrm{d} u=u(t+)-u(s+) .
$$

Definition 10. [10] Let $C:\left[t_{0}, T\right] \rightarrow \mathcal{C}(H)$ be a set-valued mapping whose values are nonempty closed and convex. The function $u \in B V\left(\left[t_{0}, T\right], H\right)$ will be said to be a solution of the sweeping process in the sense of "differential measures" if there exists (non uniquely) a non-negative real measure $\mu$, as well as a function $u^{\prime} \in \mathcal{L}_{\text {loc }}^{1}\left(\left[t_{0}, T\right] ; H\right)$ such that $\mathrm{d} u=u^{\prime} \mu$ and

$$
\forall t \in\left[t_{0}, T\right], \quad-u^{\prime}(t) \in \partial I_{\mathcal{C}(t)}[u(t)] .
$$

Proposition 15. [10] Let $C:\left[t_{0}, T\right] \rightarrow \mathcal{C}(H)$ be a set-valued mapping with nonempty closed convex values, and $u_{1}, u_{2} \in B V\left(\left[t_{0}, T\right], H\right)$ be two solutions in the sense of differential measures of the associated sweeping process. These two solutions are assumed to be both right-continuous, and to agree with the same initial condition $u_{1}\left(t_{0}\right)=u_{2}\left(t_{0}\right)=a$. Then

$$
\forall t \in\left[t_{0}, T\right], \quad u_{1}(t)=u_{2}(t) .
$$

Theorem 10. [10] Let $C:\left[t_{0}, T\right] \rightarrow \mathcal{C}(H)$ be a set-valued mapping with nonempty closed convex values and which is assumed to have bounded right-continuous retraction. Then every weak solution of the associated sweeping process (which is a function with bounded variation by virtue of Theorem 8 and right-continuous by virtue of Propositions 6 and 11) will also be a solution in the sense of differential measures.

If, in addition, $C:\left[t_{0}, T\right] \rightarrow \mathcal{C}(H)$ is assumed to show absolutely continuous retraction, then every weak solution will be a strong solution in the sense

$$
\text { for a.a. } t \in\left[t_{0}, T\right], \quad-u^{\prime}(t) \in \partial I_{\mathcal{C}(t)}[u(t)] .
$$

\section{References}

1. Adams, D.R.: The biharmonic obstacle problem with varying obstacles and a related maximal operator. Oper. Theory 110, 1-12 (1999)

2. ANDERSSON, L.E.: Existence result for quasistatic contact problem with Coulomb friction. Appl. Math. Optim. 42, 169-202 (2000)

3. Ballard, P.: A counter-example to uniqueness in quasi-static elastic contact problems with friction. Int. J. Eng. Sci. 37, 163-178 (1999)

4. Beer, G.: A Polish topology for the closed subsets of a Polish space. Proc. Am. Math. Soc. 113, 1123-1133 (1991)

5. Beer, G.: Wijsman convergence: a survey. Set-Valued Anal. 2, 77-94 (1994)

6. EcK, C., JARUŠEK, J., KRBEC, M.: Unilateral Contact Problems in Mechanics. Variational Methods and Existence Theorems. Monographs and Textbooks in Pure and Appl. Math. No. 270. Chapman \& Hall/CRC, New York (2005)

7. JARUŠEK, J.: Contact problems with bounded friction. Czechoslov. Math. Jo. 33, 108, 237-261 (1983) 
8. Kinderlehrer, D., Stampacchia, G.: An Introduction to Variational Inequalities and their Applications. Academic Press, New York, 1980

9. Moreau, J.J.: Multiapplications à rétraction finie. Annali della Scuola Normale Superiore di Pisa 1, 169-203 (1974)

10. Moreau, J.J.: Evolution problem associated with a moving convex set in a Hilbert space. J. Differ. Equ. 26, 347-374 (1977)

11. Suquet, P.M.: Discontinuities and plasticity. Nonsmooth Mechanics and Applications (Eds. J.J. Moreau and P.D. Panagiotopoulos), cIsM Courses No 302. Springer, Berlin, 279-341 (1988)

12. Rockfellar, R.T., Wets, R.J-B.: Variational Analysis. Springer, Berlin, 1998

13. Yosida, K.: Functional Analysis, 6th edn. Springer, Berlin (1980) 